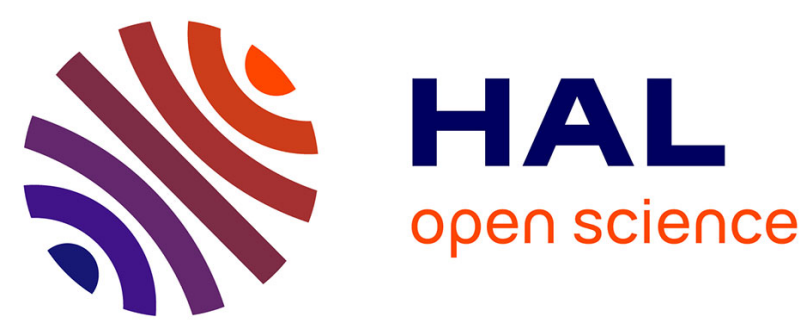

\title{
Synthetic studies towards
}

4,10-diaza-1,7-dioxaspiro[5.5]-undecanes: access to 3-aza-6,8 dioxabicyclo[3.2.1] octan-2-one and $2 \mathrm{H}-1,4$-oxazin-3(4H)-one frameworks.

Marlène Goubert, Loic Toupet, Marie-Eve Sinibaldi, Isabelle Canet

\section{To cite this version:}

Marlène Goubert, Loic Toupet, Marie-Eve Sinibaldi, Isabelle Canet. Synthetic studies towards 4,10-diaza-1,7-dioxaspiro[5.5]-undecanes: access to 3-aza-6,8 dioxabicyclo[3.2.1]octan-2-one and 2H1,4-oxazin-3(4H)-one frameworks.. Tetrahedron, 2007, 63, pp.8255-8266. 10.1016/j.tet.2007.05.109 . hal-00186417

\section{HAL Id: hal-00186417 https://hal.science/hal-00186417}

Submitted on 9 Nov 2007

HAL is a multi-disciplinary open access archive for the deposit and dissemination of scientific research documents, whether they are published or not. The documents may come from teaching and research institutions in France or abroad, or from public or private research centers.
L'archive ouverte pluridisciplinaire HAL, est destinée au dépôt et à la diffusion de documents scientifiques de niveau recherche, publiés ou non, émanant des établissements d'enseignement et de recherche français ou étrangers, des laboratoires publics ou privés. 


\title{
Synthetic studies towards 4,10-diaza-1,7-dioxaspiro[5.5]- undecanes: access to 3-aza-6,8-dioxabicyclo[3.2.1]octan-2-one and $2 H-1,4-0 x a z i n-3(4 H)$-one frameworks
}

\author{
Marlène Goubert, ${ }^{\mathrm{a}}$ Loïc Toupet, ${ }^{\mathrm{b}}$ Marie-Eve Sinibaldi ${ }^{\mathrm{a}, *}$ and Isabelle Canet ${ }^{\mathrm{a}, *}$ \\ ${ }^{a}$ Laboratoire de Synthèse Et Etude de Systèmes à Intérêt Biologique, UMR CNRS 6504, Université Blaise Pascal, \\ 63177 Aubière Cedex, France \\ ${ }^{\mathrm{b}}$ Groupe Matière Condensée et Matériaux, UMR CNRS 6626, Université de Rennes 1, 35042 Rennes Cedex, France
}

\begin{abstract}
Synthetic approaches towards 4,10-diaza-1,7-dioxaspiro[5.5]undecanes starting from 1,3-dichloroacetone and solketal derivatives are explored. The method relies on the preparation of a key bis-substituted dihydroxy-protected oxime, which would undergo a final acidic deprotection-spiroacetalization process. Although the desired diazaspiroketal framework could not be obtained, our conditions led to the unexpected 3-aza-6,8-dioxabicyclo[3.2.1]octan-2-one $\mathbf{1 8}$ or to the oxazinone $\mathbf{3 2}$ in good yields.
\end{abstract}

\section{Introduction}

Molecules owning a spiroketal core are abundant as natural products and many of them exhibit important biological properties. ${ }^{1}$ Moreover, the rigid spiroketal framework possesses strong conformational preferences ${ }^{2}$ and therefore could be used as structural scaffolds for binding to a receptor. Consequently, there is sustained interest in the synthesis of these moieties and/or substituted analogues. Recently, new spiroketals incorporating nitrogen in their cycles, presenting emphasis in activities of their unsubstituted analogues, have been described in the literature, such as new antifeedant Tonghaosu analogue, ${ }^{3}$ GD3-lactam ligand used in the development of an anti-melanoma vaccine ${ }^{4}$ and tachykinin antagonists ${ }^{5}$ (Fig. 1). Additionally, spirocyclic ketallactone frameworks have been designed as novel structures amenable to combinatorial prospecting libraries. ${ }^{6}$

Thus, the structural novelty and the biological relevance of 4- and/or 10-aza-1,7-dioxaspiro[5.5]undecane class of compounds suggested to develop synthetic pathways to their skeleton.

In a previous work, ${ }^{7}$ we disclosed an efficient two-step procedure for the preparation of 4,10-dioxa- or 4,10-dithiaspiroketals 1. Our method was based on an acidic one-pot deprotection-spirocyclization process of a key protected

* Corresponding authors. Tel.: +33473 405 284; fax: +33473407 717 (M.-E.S.); tel.: +33 473407875 ; fax: +33473407 717 (I.C.); e-mail addresses: m-eve.sinibaldi-troin@univ-bpclermont.fr; isabelle.canet@ univ-bpclemont.fr ketone $\mathbf{5}$, issued from a double substitution of the dichlorooxime $\mathbf{4}$ by the alcohol $\mathbf{3 a}$ or its thiol derivative $\mathbf{3 b}$, promoted by $\mathrm{KH}$ in THF (Scheme 1).

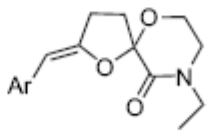

Tonghaosu analogue<smiles>[Al]C1CCC2(OCCN[C@H]2[Al])OC1</smiles>

NK1 antagonists

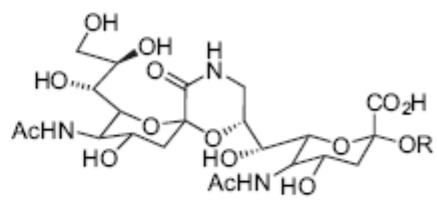

analogues of ganglioside : GD3-lactam

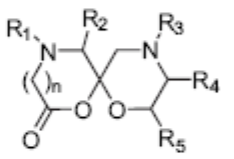

skeleton of ring systems assembled on solid phase
Figure 1.

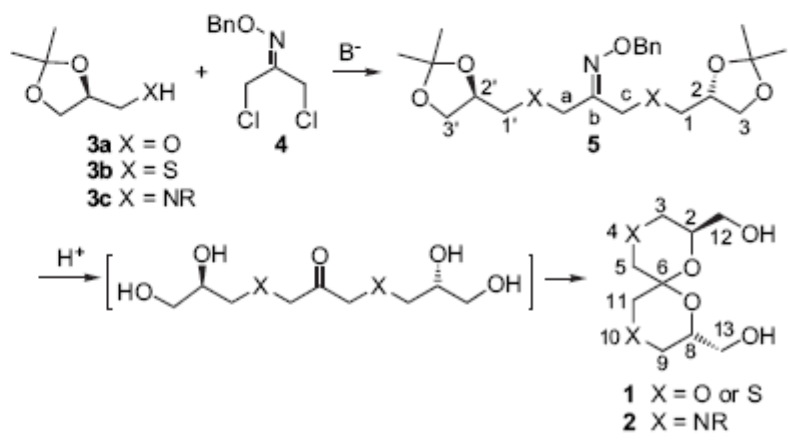

Scheme 1. 
We then wished to extend our pathway and focussed our attention to the synthesis of aza derivatives such as 4,10-diaza1,7-dioxaspiro[5.5] undecane skeleton.

To this purpose, we reported here our explorating studies towards structure $2(X=N R)$, using oxime 4 and solketal derivatives $3 \mathbf{c}$ as starting materials (Scheme 1).

\section{Results and discussion}

\subsection{Approaches to the 4,10-diaza-1,7-dioxaspiro[5.5]- undecane framew ork}

We first investigated the alkylation of amines $( \pm)-6^{8}$ and $( \pm)-7^{9}$ with oxime 4 in basic media (Scheme 2). Surprisingly, treatment of 4 by $\mathbf{6}$ alone or in the presence of a mineral base such as $\mathrm{K}_{2} \mathrm{CO}_{3}, \mathrm{Cs}_{2} \mathrm{CO}_{3}$, CsOH or $\mathrm{KOH} / 18$-crown-6, led invariably to polycondensation of starting oxime 4 accompanied with numerous by-products. Modifying the temperature or the nature of the solvent did not give better results. At last, oxime $8^{10}$ could be conveniently obtained in a $77 \%$ yield by the condensation of 4 with an excess of 7 in refluxing methanol.

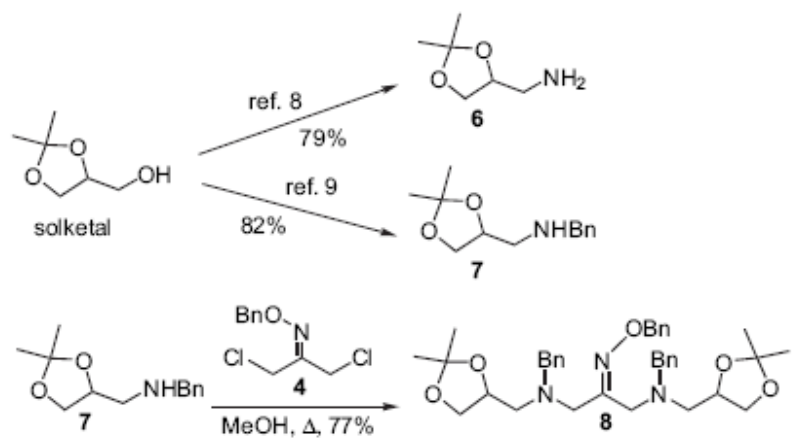

Scheme 2.

The final step of our synthetic scheme involved deprotection of both ketone and alcohol functions of $\mathbf{8}$ and subsequent acid-catalyzed cyclization. This sequence was undertaken under our preliminary optimized conditions, Amberlyst ${ }^{\circledR}$ 15 in acetone $/ \mathrm{H}_{2} \mathrm{O}$ with or without paraformaldehyde. ${ }^{7}$
Aza compound 8 behaved differently than its oxa- or thiaanalogues and remained unchanged. We therefore next tested other conditions by varying the solvent and the acidic medium, namely using $\mathrm{HCl} 5 \%$ in THF, $\mathrm{Zn}$ powder in $\mathrm{AcOH},{ }^{11} \mathrm{SnCl}_{2} \cdot 2 \mathrm{H}_{2} \mathrm{O} / \mathrm{SiO}_{2}$ in $\mathrm{THF}^{12}$ or $\mathrm{CeCl}_{3} \cdot 7 \mathrm{H}_{2} \mathrm{O}$ / $(\mathrm{COOH})_{2}$ in $\mathrm{CH}_{3} \mathrm{CN} .{ }^{13}$ Once again, no spiroketal was detected in the reaction mixture. All these attempts led either to degradation products or to the sole deprotection of the diol functions, leaving the $O$-benzyloxime group unchanged. Heating 8 with $\mathrm{HCl}$ and formaldehyde in THF during 3 days, led to traces of a compound owning the expected formula $\mathrm{C}_{23} \mathrm{H}_{30} \mathrm{~N}_{2} \mathrm{O}_{4}$ (detected in the crude reaction mixture by mass spectrometry, $[\mathrm{M}+\mathrm{H}]^{+}$at $m / z=399$ ). Anyway, as we were unable to isolate a pure scale of it, we could not characterize this new compound.

To circumvent these problems, we chose to protect the amino groups as amides or as a carbamate. To this end, we modified our synthetic approach and prepared solketal derivatives $( \pm)-9 \mathbf{a}$ and $( \pm)-9 \mathbf{b}$ to examine their condensation on dichlorooxime 4 in basic media (Scheme 3). Amides 9a,b did not afford the expected bis-substituted oximes 10a,b. In fact, whatever the involved basic conditions, partial dimerization or decomposition ${ }^{14}$ of oxime 4 was observed, all starting amides remaining unchanged. In the case of carbamate ( \pm )-9c, only the use of $\mathrm{KH}$ (3.0 equiv) in THF at $20^{\circ} \mathrm{C}$ afforded cleanly the monosubstituted oxime $( \pm)-11$, which could be isolated in an improved 33\% yield. Extending the reaction time or heating the reaction mixture damaged the starting materials (Scheme 3).

Pursuing our aim, we next envisaged another route to oximes $10 a, c$, introducing this time the nitrogen atom on the starting oxime 4 .

We thus synthesized the diamine 12 readily available from 4 in two steps. ${ }^{15}$ Bis-acylation of $\mathbf{1 2}$ furnished efficiently the expected diamide $13 \mathbf{a}$ or dicarbamate $13 \mathbf{b}$ precursors. These compounds were then engaged in a condensation with the previously described iodide $( \pm)-\mathbf{1 4}{ }^{16}$ in order to obtain oximes 10a,c. Unfortunately, when 13a,b were subjected to treatment by $n$-BuLi or LDA in THF followed by the addition of $\mathbf{1 4}$, no reaction occurred. Changing the base ( $\mathrm{KH}$ instead of lithiated bases) led to the degradation of both starting materials (Scheme 4).

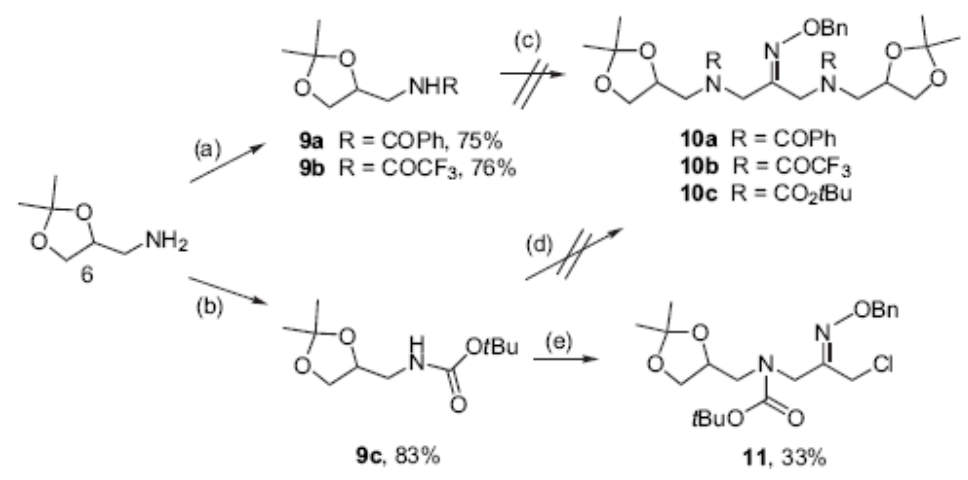

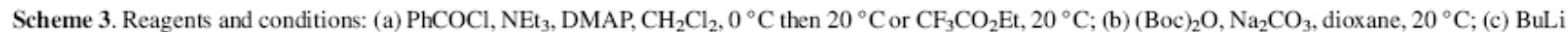
or KH, THF; (d) BuLi or $t$-BuOK, THF; (e) KH, THF then $4,20^{\circ} \mathrm{C}$. 


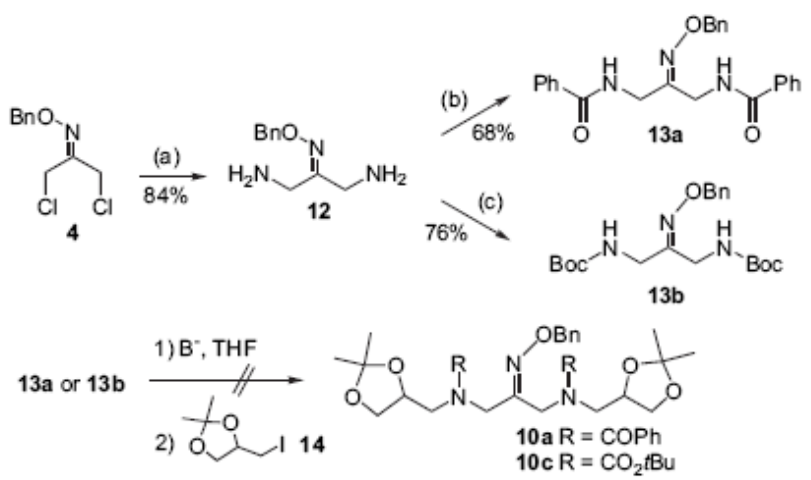

Scheme 4. Reagents and conditions: (a) PhtNK, DMF, reflux then $\mathrm{NH}_{2}$ $\mathrm{NH}_{2}, \mathrm{MeOH}$, reflux; (b) $\mathrm{PhCOCl}, \mathrm{NEt}_{3}, \mathrm{DMAP}, \mathrm{CH}_{2} \mathrm{Cl}_{2}, 0^{\circ} \mathrm{C}$ then $20^{\circ} \mathrm{C}$; (c) $(\mathrm{Boc})_{2} \mathrm{O}, \mathrm{Na}_{2} \mathrm{CO}_{3}$, dioxane, $20^{\circ} \mathrm{C}$.

\subsection{Approaches to the 4,10-diaza-1,7-dioxa-3,9-dioxo- spiro[5.5] undecane framework}

Once again, we reoriented our synthetic plan to diazaspiroketals and developed an alternative route based upon the preparation of the bis-substituted oxime 16, possessing now the required amide groups in its linear chain.

Thus, bis-condensation of the crude diamine 12 with acylchloride $(R)-15^{17}$ in the presence of triethylamine and a catalytic amount of 4-dimethylaminopyridine in dichloromethane, furnished the oxime $(R, R)-16$ in a $59 \%$ overall yield from $(S)$-solketal (Scheme 5). Deprotection of both diol and ketone functions was carried out using Amberlyst ${ }^{\circledR} 15$ in acetone/water $(10 / 1)$ at reflux and gave the key intermediate

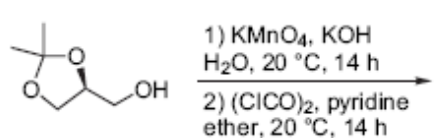

(S)-solketal

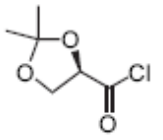

(R)-15<smiles></smiles>
12 $(R, R)-16$

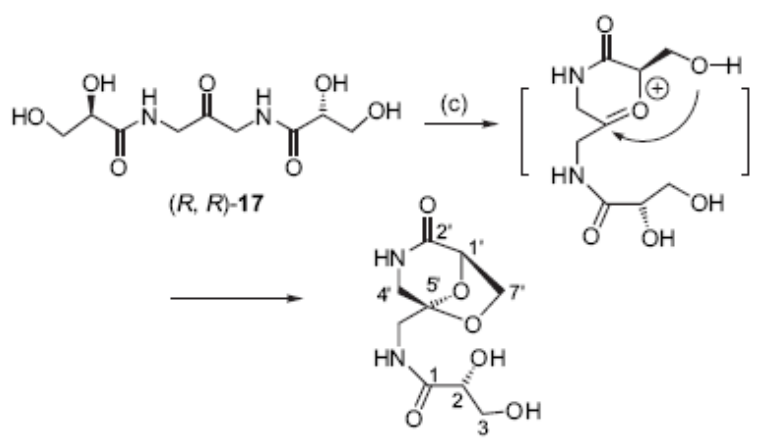

$(2 R, 6 R, 8 R)-18$

Scheme 5. Reagents and conditions: (a) $\mathrm{NEt}_{3}, \mathrm{DMAP}, \mathrm{CH}_{2} \mathrm{Cl}_{2},(R)-15$, $67 \%$; (b) Amberlyst ${ }^{\infty} 15$, acetone $/ \mathrm{H}_{2} \mathrm{O},(10 / 1), \Delta, 77 \%$; (c) $p \mathrm{TsOH}, n$-butanol, $\Delta, 6 \mathrm{~h}, 76 \%$ after recrystallization.
$(R, R)-(17)$ in a $77 \%$ yield. Contrarily to the oxa- and thia-derivatives, ${ }^{7}$ these mild acidic conditions were insufficient to promote the ultimate spiroacetalization. So, we engaged oxime 17 in refluxing $n$-butanol in the presence of a catalytic amount of para-toluenesulfonic acid. After $6 \mathrm{~h}$, we observed the exclusive formation of the unexpected bicyclic lactam 18 , which was isolated in a nearly quantitative yield $(76 \%$ after recrystallization) (Scheme 5). The formation of 18 resulted from the nucleophilic attack of the hydroxymethyl group of the cycle on the oxonium intermediate. ${ }^{18}$ This attack appeared indeed more favourable than the attack of the secondary alcohol — which could lead to a spiroketal—as this latter required a two-step process including the isomerization of the trans amide function prior to spiroacetalization.

The NMR data of 18 were in good agreement with the indicated structure. The chemical shift of the deshielded $\mathrm{C}-5^{\prime}$ at $\delta=104.5 \mathrm{ppm}$, together with the vicinal ${ }^{3} J_{\mathrm{H}, \mathrm{H}}$ couplings (i) between $\mathrm{C}_{2}-\mathrm{O}-\mathrm{H}(\delta=5.59 \mathrm{ppm})$ and $\mathrm{H}-2(\delta=3.91 \mathrm{ppm})$ and (ii) between $\mathrm{H}-3 \mathrm{a}(\delta=3.57 \mathrm{ppm})$ or $\mathrm{H}-3 \mathrm{~b}(\delta=3.46 \mathrm{ppm})$ and $\mathrm{C}_{3}-\mathrm{O}-\mathrm{H}(\delta=4.72 \mathrm{ppm})$, confirmed a bridged structure for 18. The formation of this bicycle was also corroborated by the presence of a strong correlation peak between $\mathrm{C}-5^{\prime}$ and $\mathrm{H}-7 \mathrm{a}^{\prime}$ on the HMBC spectrum.

The $(S)$ configuration of the starting solketal imposed $(R)$ configurations for $\mathrm{C}-2$ and $\mathrm{C}-\mathrm{1}^{\prime}$ in 18 . Since C-5' could only adopt an $(R)$ configuration in the intramolecular acetalization process, compound 18 possesses then a $\left(1^{\prime} R, 2 R, 5^{\prime} R\right)$ configuration.

Compound 18 occurred as a fine powder and could be recrystallized from ethanol; we then confirmed its structure by Xray crystallographic analysis. The ORTEP shown in Figure 2 exhibits a $\left(5^{\prime} R\right)$ configuration and a trans conformation of the amide in the lateral chain in $\mathbf{1 8}$

To avoid this competitive cyclization, we decided to selectively protect the two primary hydroxyl groups of 17 . Unfortunately, the polarity and the low solubility of compound $\mathbf{1 7}$ in classical solvent did not permit to carry out efficiently this protection. So we planed to perform it on the partially deprotected oxime 21 , quantitatively prepared by refluxing 16 with a catalytic amount of $\mathrm{D}, \mathrm{L}-\mathrm{c}$-amphorsulfonic acid in ethanol for $3 \mathrm{~h}$ (Scheme 6). Treatment of $\mathbf{2 1}$ with dibutyltin oxide in toluene/methanol $(10: 1, \mathrm{v} / \mathrm{v})$ followed by the addition of benzylbromide and tetrabutylammonium iodide ${ }^{19}$ led to 22a in a $34 \%$ yield. Removal of the oxime group was realized using Amberlyst ${ }^{\circledR} 15$ in refluxing acetone/water $(10: 1, \mathrm{v} / \mathrm{v})$ and afforded ketone $\mathbf{2 3}$ in a $85 \%$ yield. Unfortunately, treatment of $\mathbf{2 3}$ under the same cyclization conditions as that for 17 ( $p$ TsOH in $n$-butanol) led to the loss of the protective groups and gave once again the bicyclic compound 18. Attempts to prepare the protected di-TBDPS compound from 21 failed. Only the monosilylether derivative $\mathbf{2 2 b}$ was formed, in a $33 \%$ yield. No intramolecular TBDPS transfer was observed in our conditions.

At this stage, we envisaged the reaction of the diamine 12 on the acylchloride 27 possessing two alcohol functions orthogonally protected. The choice of the protective groups of template 27 was now crucial. Because of our condensation and spirocyclization conditions, we chose a $\mathrm{MOM}^{20}$ protective 

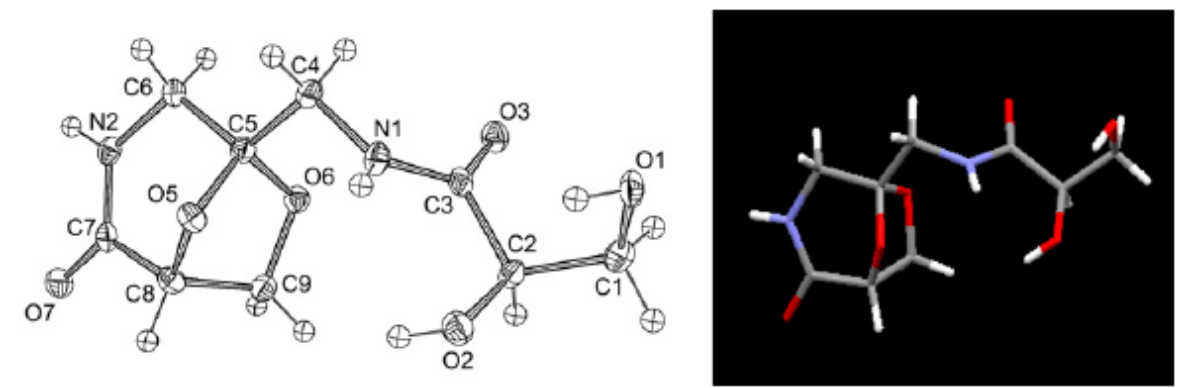

Figure 2. ORTEP drawing of 18 .

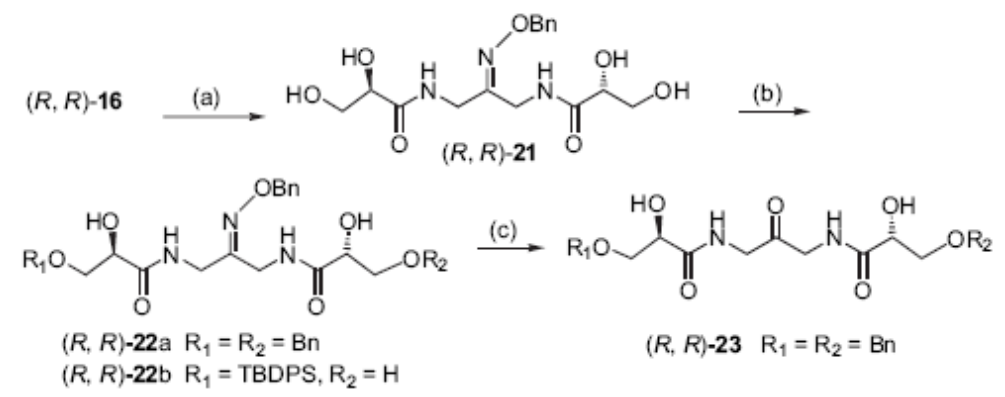

Scheme 6. Reagents and conditions: (a) $\mathrm{CSA}$, EtOH, $\Delta, 3 \mathrm{~h}$, quant; (b) (i) $\mathrm{Bu}_{2} \mathrm{SnO}$, toluene/MeOH, (10/1), $\Delta, 5-6 \mathrm{~h},(\mathrm{ii}) \mathrm{BnBr}, \mathrm{Bu} 4 \mathrm{NI}, \Delta, 4-5 \mathrm{~h}, 34 \%$ in two steps for $22 \mathrm{a}$ or imidazole, TBDPSCl, DMF, $0^{\circ} \mathrm{C}$ then $20^{\circ} \mathrm{C}, 14 \mathrm{~h}, 33 \%$ for $22 \mathrm{~b}$; (c) Amberlyst ${ }^{\infty} 15$, acetone/ $\mathrm{H}_{2} \mathrm{O},(10 / 1), \Delta, 85 \%$.

group for the secondary alcohol and a TBDPS protective group for the primary one. Thus, compound $(R)-27$ was prepared, in a six-step sequence and a $58 \%$ overall yield, starting from D-serine (Scheme 7).

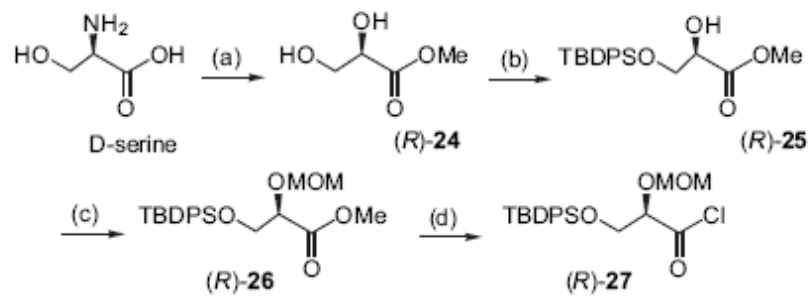

Scheme 7. Reagents and conditions: (a) (i) $\mathrm{NaNO}_{2}, \mathrm{H}_{2} \mathrm{SO}_{4}, \mathrm{H}_{2} \mathrm{O}$, (ii) $\mathrm{HC}(\mathrm{OMe})_{3}, \mathrm{H}_{2} \mathrm{SO}_{4}, \mathrm{MeOH}, 60{ }^{\circ} \mathrm{C}, 30 \mathrm{~min}, 83 \%$ (Ref. 21); (b) imidazole, $\mathrm{CH}_{2} \mathrm{Cl}_{2}$, TBDPSCl, $-40^{\circ} \mathrm{C}, 1 \mathrm{~h} 30 \mathrm{~min}, 83 \%$ (Ref. 22 ); (c) MOMCl, $(i$ $\mathrm{Pr})_{2} \mathrm{EtN}, \mathrm{CH}_{2} \mathrm{Cl}_{2}, 20^{\circ} \mathrm{C}, 24 \mathrm{~h}, 84 \%$; (d) (i) $\mathrm{LiOH}, 1 \mathrm{M}$, THF/MeOH (4/1), $20{ }^{\circ} \mathrm{C}, 4 \mathrm{~h}$, (Ref. 23) (ii) $(\mathrm{ClCO})_{2}$, pyridine, $\mathrm{Et}_{2} \mathrm{O}, 20^{\circ} \mathrm{C}, 14 \mathrm{~h}$, quant.

Condensation of crude 12 with undistilled $(R)-27$ gave the bis-substituted oxime 28 in a good yield of $37 \%$. The further one-step cleavage of both MOM-ether and oxime groups revealed to be, in fact, not so trivial. Treatment of 28 with Amberlyst ${ }^{\circledR} 15$ and paraformaldehyde in refluxing acetone $/ \mathrm{H}_{2} \mathrm{O}$ $(10: 1, \mathrm{v} / \mathrm{v})$, afforded partially deprotected ketone $(R, R)-\mathbf{3 0 a}$ in a low yield of $26 \%$, accompanied with numerous nonpolar side products we did not characterize.

To complete the deprotection, we developed a sequential process for the removal of the different protective groups. The best results were obtained starting by the cleavage of the MOM groups of $\mathbf{2 8}$ according to a literature procedure. ${ }^{24}$ Action of trimethylsilylbromide in $\mathrm{CH}_{2} \mathrm{Cl}_{2}$ at $0{ }^{\circ} \mathrm{C}$ for $4 \mathrm{~h}$ led as expected to the diol 29, accompanied with the ketone 30b (Scheme 8). Fortunately, in our case, the potentially problematic migration of the TBDPS group to the adjacent oxygen atom was not observed under our reaction conditions. Increasing the temperature or the duration of the reaction did not improve the yield of $30 \mathrm{~b}$ but damaged the products. At this stage oxime 29 and ketone $30 \mathrm{~b}$ could be easily separated and fully characterized.

Oxime 29 was then re-engaged in classical cleavage conditions (Amberlyst ${ }^{\circledR}$ 15/paraformaldehyde in refluxing acetone $\left./ \mathrm{H}_{2} \mathrm{O}(10: 1, \mathrm{v} / \mathrm{v})\right)$ and was transformed into ketone $30 \mathrm{~b}$, but in a modest yield of $26 \%$ (see Scheme 8 ). However, this deprotection-reaction step was clean and oxime 29 could be recycled after column chromatography separation.

We last attempted the isomerization-spiroacetalization of the ketodiol $30 \mathrm{~b}$ in various acidic media. Using Amberlyst $\left.{ }^{(}\right) 15$ in acetone/ $\mathrm{H}_{2} \mathrm{O}(10 / 1, \mathrm{v} / \mathrm{v}), \mathrm{Yb}(\mathrm{OTf})_{3}$ in $\mathrm{CH}_{3} \mathrm{CN}, \mathrm{BF}_{3} \cdot \mathrm{Et}_{2} \mathrm{O}$ in THF or $p$ TsOH in THF, no reaction occurred. The use of $p$ TsOH in $n$-butanol furnished compound 31. As for compound 17 , the trans configuration of the amide group in the lateral chain disfavoured the intramolecular final spirocyclization for the benefit of an intermolecular reaction of the oxonium intermediate with the butanol, which acted as a nucleophile. Treating $30 \mathrm{~b}$ with $p \mathrm{TsOH}$ for $7 \mathrm{~h}$ in refluxing toluene or boiling $\mathbf{3 1}$ in toluene led to the same oxazinone $\mathbf{3 2}$, which resulted, in the case of $\mathbf{3 1}$, from a classical elimination of butanol in the acid medium (Scheme 8).

Since access to spiroketals by treatment of 2-substituted dihydropyrane in various acidic media (PPTS, ${ }^{25}$ $\mathrm{BF}_{3} \cdot \mathrm{Et}_{2} \mathrm{O},{ }^{25 \mathrm{~b}} \mathrm{D}, \mathrm{L}-\mathrm{camphorsulfonic}$ acid $^{25 \mathrm{c}}$ ) has been already described, we decided to apply these conditions to the oxazinone 32. Unfortunately, we never detected the formation of the desired spiroheterocycle in the reaction mixtures but observed only degradation of the starting materials. 


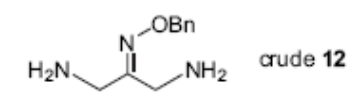

(a)

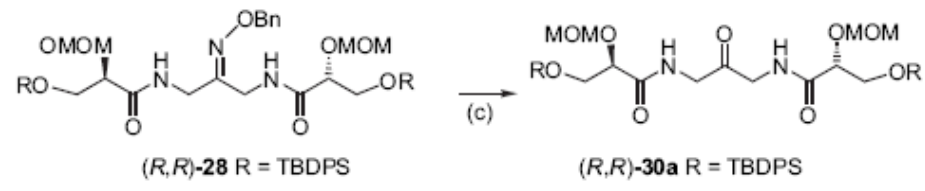

(b)<smiles>[R]OC[C@@H](O)C(=O)NCC(=O)NCC(=O)NCC(O)C(O)COc1ccccc1</smiles>

$(R, R)-29 \mathrm{R}=\mathrm{TBDPS} \quad(R, R)-30 \mathrm{~b} \mathrm{R}=\mathrm{TBDPS}$

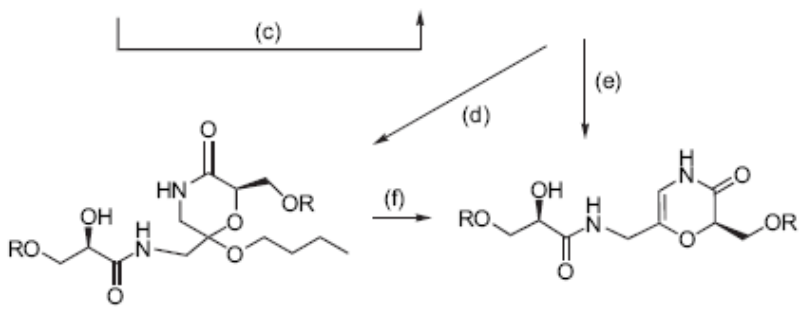

$(R, R)-31 \mathrm{R}=\mathrm{TBDPS}$

$(R, R)-32 \mathrm{R}=\mathrm{TBDPS}$

Scheme 8. Reagents and conditions: (a) NEt, DMAP, $\mathrm{CH}_{2} \mathrm{Cl}_{2},(R)-27,37 \%$; (b) TMSBr$, \mathrm{CH}_{2} \mathrm{Cl}_{2}, 0{ }^{\circ} \mathrm{C}, 4 \mathrm{~h},((R, R)-2954 \%$ and $(R, R)-30 \mathrm{~b} 20 \%$ after purification); (c) Amberlyst ${ }^{\infty} 15,\left(\mathrm{CH}_{2} \mathrm{O}\right)_{n}$, acetone/ $\mathrm{H}_{2} \mathrm{O}(10 / 1), \Delta, 24 \mathrm{~h}, 26 \%$; (d) 0.04 equiv, $p \mathrm{TsOH}, n$-butanol, $\Delta, 6 \mathrm{~h}$; (e) 0.04 equiv, $p$ TsOH, toluene, $\Delta, 7 \mathrm{~h}$, $57 \%$; (f) toluene, $\Delta, 5 \mathrm{~h}, 57 \%$ in two steps from $(R, R)-30 \mathrm{~b}$

\section{Conclusion}

In this paper, we described our preliminary work towards a novel rigid spiroketal framework incorporating one nitrogen in each cycle.

Our strategy underlied first upon the condensation of $(S)$-solketal derivatives on dichloroacetone $O$-benzyloxime. Direct approaches to 4,10-diaza-1,7-dioxaspiro[5.5] undecane core were unsuccessful and showed the necessity of using amide functions as integral parts of the skeleton of the molecule instead of protective groups.

Using this second approach we prepared oximes 17 and 28 . However, we were unable to achieve the final spiroacetalization of $\mathbf{1 7}$ and $\mathbf{2 8}$, illustrating the difficulties in preparing the 4,10-diazaspiroketal compounds.

Meanwhile, we synthesized the new 3-aza-6,8-dioxabicyclo[3.2.1]octan-2-one $\left(1^{\prime} R, 2 R, 5^{\prime} R\right)-18$ in six steps in a $31 \%$ overall yield from dichloroacetone, through an unusual intramolecular dehydration reaction.

The same protocol led to the original oxazinone 32 isolated in nine steps and $2 \%$ overall yield starting from D-serine.

In addition, the achievement of the monosubstituted chlorooxime 11 allowed us to envisage an effective access to original 'dissymmetrical heteroatom' 4,10-disubstituted spiroketals. These investigations are currently in progress in our laboratory and will be published in due course.

\section{Experimental}

\subsection{General}

Melting points were measured using a Reichert melting point apparatus and are uncorrected. Infra Red spectra were recorded on a Perkin-Elmer 881 instrument. ${ }^{1} \mathrm{H}$ NMR $(400 \mathrm{MHz})$ and ${ }^{13} \mathrm{C}$ NMR $(100 \mathrm{MHz})$ were recorded with a Bruker AC 400 spectrometer. Chemical shifts ( $\delta$ values) are expressed in parts per million (ppm) and coupling constants $(J)$ are expressed in Hertz. NMR spectra were recorded in $\mathrm{CDCl}_{3}, \mathrm{CD}_{3} \mathrm{OD}$ or DMSO- $d_{6}$, using the solvent signals as reference. Mass spectra were recorded with a Hewlett Packard 5989B instrument and high-resolution mass spectra (HRMS) were performed with a Q-TOF micromass. Elemental analysis was performed using an elemental analyzer. Optical rotations were measured at sodium D-line $(589 \mathrm{~nm})$ using a $1 \mathrm{dm}$ quartz cell with a Jasco DIP-370 apparatus. Chromatography was performed using silica gel 60 (230400 mesh) and thin layer chromatography (TLC) was performed on silica gel $60 \mathrm{PF}_{254}$ plates $(20 \times 20 \mathrm{~cm})$. Compounds were identified using UV fluorescence $(\lambda=254 \mathrm{~nm})$ and/or staining with a $5 \%$ phosphomolibdic acid solution in ethanol following by heating. Commercially reagents (Aldrich, Acros, Lancaster) were used as received without additional 
purification. Tetrahydrofuran (THF) was distilled from potassium/benzophenone while dichloromethane $\left(\mathrm{CH}_{2} \mathrm{Cl}_{2}\right)$ was dried over calcium hydride prior to use. Suitable crystal for structure determination was obtained by crystallization from ethanol. Crystal was obtained with a diffractometer Oxford Diffraction Xcalibur Saphir 3 at the University of Rennes I by Loïc Toupet.

\subsection{Synthesis}

4.2.1. 1,3-Dichloropropan-2-one $\boldsymbol{O}$-benzyloxime (4). 1,3Dichloroacetone $(1.27 \mathrm{~g}, 10 \mathrm{mmol})$ was added to a solution of benzylhydroxylamine hydrochloride $(1.60 \mathrm{~g}, 10 \mathrm{mmol})$ in ethanol $(15 \mathrm{~mL})$. The mixture was stirred at $20^{\circ} \mathrm{C}$ for $24 \mathrm{~h}$. The solvent was removed and the residue was treated three times with ethanol. Then cyclohexane $(150 \mathrm{~mL})$ was added before drying over $\mathrm{MgSO}_{4}$. The precipitate was filtered off. The filtrate was concentrated to give quantitatively 4 as a colourless liquid (2.32 g). ${ }^{1} \mathrm{H}$ NMR (400 MHz, $\mathrm{CDCl}_{3}$ ): $\delta 7.40-7.30(\mathrm{~m}, 5 \mathrm{H}, \mathrm{Ph}), 5.18\left(\mathrm{~s}, 2 \mathrm{H}, \mathrm{CH}_{2} \mathrm{Ph}\right), 4.38(\mathrm{~s}, 2 \mathrm{H}$, $\left.\mathrm{CH}_{2} \mathrm{Cl}\right), 4.28\left(\mathrm{~s}, 2 \mathrm{H}, \mathrm{CH}_{2} \mathrm{Cl}\right) ;{ }^{13} \mathrm{C} \mathrm{NMR}(100 \mathrm{MHz}$, $\left.\mathrm{CDCl}_{3}\right): \delta 151.4(\mathrm{C}=\mathrm{N}), 136.7(\mathrm{C}-\mathrm{Ar}), 128.5(\mathrm{C}-\mathrm{Ar})$, $128.2(\mathrm{C}-\mathrm{Ar}), 128.1(\mathrm{C}-\mathrm{Ar}), 76.9\left(\mathrm{CH}_{2} \mathrm{Ph}\right), 42.1\left(\mathrm{CH}_{2} \mathrm{Cl}\right)$, $32.8\left(\mathrm{CH}_{2} \mathrm{Cl}\right)$.

4.2.2. ( \pm )-(2,2-Dimethyl-1,3-dioxolan-4-yl)methanamine (6). To a solution of solketal $(3.0 \mathrm{~g}, 22.8 \mathrm{mmol})$ in anhydrous THF $(230 \mathrm{~mL})$ were added successively triphenylphosphine $(7.2 \mathrm{~g}, 27.3 \mathrm{mmol})$, phthalimide $(3.4 \mathrm{~g}, 22.8 \mathrm{mmol})$ and diisopropyl azodicarboxylate $(5.4 \mathrm{~mL}, 27.3 \mathrm{mmol})$. The resulting mixture was stirred $20 \mathrm{~h}$ at $20^{\circ} \mathrm{C}$ under inert atmosphere. After evaporation of the solvent under reduced pressure, flash column chromatography on silica gel with cyclohexane/ethyl acetate $(7: 3, \mathrm{v} / \mathrm{v})$ as eluent gave $2-[(2,2-$ dimethyl-1,3-dioxolan-4-yl)methyl\}]-1 $H$-isoindole-1,3(2H)dione intermediate as a white solid $(5.2 \mathrm{~g}, 88 \%) . \mathrm{Mp} 76{ }^{\circ} \mathrm{C}$ (cyclohexane); $R_{f}: 0.46$ (ethyl acetate/cyclohexane=1:1); IR $(\mathrm{KBr}): \nu 1700(\mathrm{C}=\mathrm{O}) \mathrm{cm}^{-1}$; ${ }^{1} \mathrm{H}$ NMR $(400 \mathrm{MHz}$, $\left.\mathrm{CDCl}_{3}\right): \delta 7.84\left(\mathrm{dd},{ }^{3} J=5.5 \mathrm{~Hz},{ }^{4} J=3.0 \mathrm{~Hz}, 2 \mathrm{H}, \mathrm{H}-\mathrm{Ar}\right)$, 7.71 (dd, $\left.{ }^{3} J=5.5 \mathrm{~Hz},{ }^{4} J=3.0 \mathrm{~Hz}, 2 \mathrm{H}, \mathrm{H}-\mathrm{Ar}\right), 4.43$ (tt, ${ }^{3} J=6.5$ and $\left.5.5 \mathrm{~Hz}, 1 \mathrm{H}, \mathrm{CH}-\mathrm{O}\right), 4.06\left(\mathrm{dd},{ }^{2} J=8.5 \mathrm{~Hz},{ }^{3} J=\right.$ $6.0 \mathrm{~Hz}, 1 \mathrm{H}, \mathrm{CH}_{2}-\mathrm{O}$ ), 3.92 (dd, ${ }^{2} J=14.0 \mathrm{~Hz},{ }^{3} J=7.0 \mathrm{~Hz}$, $1 \mathrm{H}, \mathrm{CH}_{2}-\mathrm{N}$ ), 3.84 (dd, ${ }^{2} J=8.5 \mathrm{~Hz},{ }^{3} J=5.0 \mathrm{~Hz}, 1 \mathrm{H}, \mathrm{CH}_{2}$ O), 3.71 (dd, ${ }^{2} J=14.0 \mathrm{~Hz},{ }^{3} J=5.5 \mathrm{~Hz}, 1 \mathrm{H}, \mathrm{CH}_{2}-\mathrm{N}$ ), 1.43 (s, 3H, Me), 1.30 (s, 3H, Me); ${ }^{13} \mathrm{C}$ NMR $(100 \mathrm{MHz}$, $\left.\mathrm{CDCl}_{3}\right): \delta 168.1(\mathrm{CO}), 134.0(\mathrm{C}-\mathrm{Ar}), 131.9(\mathrm{C}-\mathrm{Ar}), 123.3$ $(\mathrm{C}-\mathrm{Ar}), 109.7\left(\mathrm{C}-\left(\mathrm{CH}_{3}\right)_{3}\right), 73.2(\mathrm{CH}-\mathrm{O}), 67.3\left(\mathrm{CH}_{2}-\mathrm{O}\right)$, $40.9\left(\mathrm{CH}_{2}-\mathrm{N}\right), 26.7\left(\mathrm{CH}_{3}\right), 25.3\left(\mathrm{CH}_{3}\right)$; MS (ESI) m/z: 284 $[\mathrm{M}+\mathrm{Na}]^{+}$.

To a suspension of this intermediate $(3.0 \mathrm{~g}, 11.5 \mathrm{mmol})$ in methanol $(115 \mathrm{~mL})$ was added hydrazine monohydrate $(1.0 \mathrm{~mL}, 20.1 \mathrm{mmol})$. The reaction mixture was heated under reflux for $4-5 \mathrm{~h}$. The white precipitate thus obtained was dissolved by adding a solution of $\mathrm{KOH}(0.9 \mathrm{~g}$, $16.1 \mathrm{mmol})$ in methanol $(20 \mathrm{~mL})$. The resulting solution was then concentrated and $\mathrm{CH}_{2} \mathrm{Cl}_{2}$ was added. After filtration, the organic layer was washed with water and dried over $\mathrm{MgSO}_{4}$. Evaporation of the solvent led to $\mathbf{6}$ as a pale yellow liquid $(1.36 \mathrm{~g}, 90 \%)$. IR (film): $\nu$ 3374, 3308, $1220-1060 \mathrm{~cm}^{-1} ;{ }^{1} \mathrm{H}$ NMR $\left(400 \mathrm{MHz}, \mathrm{CDCl}_{3}\right): \delta 4.12$ (qd, ${ }^{3} J=6.5$ and $\left.4.5 \mathrm{~Hz}, 1 \mathrm{H}, \mathrm{CH}-\mathrm{O}\right), 4.03\left(\mathrm{dd},{ }^{2} J=8.0 \mathrm{~Hz}\right.$, $\left.{ }^{3} J=6.5 \mathrm{~Hz}, 1 \mathrm{H}, \mathrm{CH}_{2}-\mathrm{O}\right), 3.66\left(\mathrm{dd},{ }^{2} J=8.0 \mathrm{~Hz},{ }^{3} J=6.5 \mathrm{~Hz}\right.$,
$1 \mathrm{H}, \mathrm{CH}_{2}-\mathrm{O}$ ), 2.83 (dd, ${ }^{2} \mathrm{~J}=13.0 \mathrm{~Hz},{ }^{3} \mathrm{~J}=4.5 \mathrm{~Hz}, 1 \mathrm{H}, \mathrm{CH}_{2}-$ $\mathrm{N}), 2.78$ (dd, $\left.{ }^{2} J=13.0 \mathrm{~Hz},{ }^{3} J=6.0 \mathrm{~Hz}, 1 \mathrm{H}, \mathrm{CH}_{2}-\mathrm{N}\right), 1.42$ (s, 3H, Me), 1.35 (s, 3H, Me), 1.26 (br s, $\left.2 \mathrm{H}, \mathrm{NH}_{2}\right) ;{ }^{13} \mathrm{C}$ NMR $\left(100 \mathrm{MHz}, \mathrm{CDCl}_{3}\right): \delta 109.0\left(\mathrm{C}-\left(\mathrm{CH}_{3}\right)_{3}\right), 77.2(\mathrm{CH}-$ O), $66.8\left(\mathrm{CH}_{2}-\mathrm{O}\right), 44.6\left(\mathrm{CH}_{2}-\mathrm{N}\right), 26.7\left(\mathrm{CH}_{3}\right), 25.2\left(\mathrm{CH}_{3}\right)$; MS (ESI) $m / z: 132[\mathrm{M}+\mathrm{H}]^{+}, 74[\mathrm{M}-\text { acetone }+\mathrm{H}]^{+}$.

4.2.3. ( \pm )- $N$-Benzyl-1-(2,2-dimethyl-[1,3]dioxolan-4-yl)methanamine (7). To a solution of solketal (2.64 g, $20.0 \mathrm{mmol})$ and triethylamine $(3.35 \mathrm{~mL}, 24.0 \mathrm{mmol})$ in $\mathrm{CH}_{2} \mathrm{Cl}_{2}(20 \mathrm{~mL})$ at $0{ }^{\circ} \mathrm{C}$ and under argon, was added dropwise a solution of methanesulfonyl chloride $(1.85 \mathrm{~mL}$, $24.0 \mathrm{mmol})$ in $\mathrm{CH}_{2} \mathrm{Cl}_{2}(8 \mathrm{~mL})$. The reaction mixture was stirred for $2 \mathrm{~h}$ and then quenched by addition of water (4 mL). The resulting solution was extracted with $\mathrm{CH}_{2} \mathrm{Cl}_{2}$ and the organic layer was washed with a saturated $\mathrm{NaHCO}_{3}$ solution and then dried $\left(\mathrm{MgSO}_{4}\right)$. After filtration, the solvent was evaporated to give quantitatively the crude mesylate derivative $(4.21 \mathrm{~g}, 20.0 \mathrm{mmol})$. It was then dissolved in acetonitrile $(55 \mathrm{~mL})$, and benzylamine $(8.70 \mathrm{~mL}, 80.0 \mathrm{mmol})$ was added. The resulting mixture was heated under reflux for 2 days. After removal of the solvent, ethyl acetate $(50 \mathrm{~mL})$ was added, followed by a saturated $\mathrm{NaHCO}_{3}$ solution $(10 \mathrm{~mL})$. The layers were separated and the organic one was washed with brine and dried $\left(\mathrm{MgSO}_{4}\right)$. After filtration and concentration, the residue was purified by flash column chromatography using ethyl acetate/cyclohexane (7:3-9:1, $\mathrm{v} / \mathrm{v})$ as eluent to give 7 as an orange liquid $(3.60 \mathrm{~g}, 82 \%)$. IR (neat): $\nu 3300,1250-1050 \mathrm{~cm}^{-1} ;{ }^{1} \mathrm{H}$ NMR $(400 \mathrm{MHz}$, $\left.\mathrm{CDCl}_{3}\right): \delta$ 7.33-7.24 (m, 5H, $\mathrm{H}-\mathrm{Ar}$ ), 4.26 (quint, $\left.{ }^{3} J=6.0 \mathrm{~Hz}, 1 \mathrm{H}, \mathrm{CH}-\mathrm{O}\right), 4.03\left(\mathrm{dd},{ }^{2} J=8.0 \mathrm{~Hz},{ }^{3} J=6.5 \mathrm{~Hz}\right.$, $\left.1 \mathrm{H}, \mathrm{CH}_{2}-\mathrm{O}\right), 3.83\left(\mathrm{~d},{ }^{2} J=13.5 \mathrm{~Hz}, 1 \mathrm{H}, \mathrm{CH}_{2} \mathrm{Ph}\right), 3.82$ (d, $\left.{ }^{2} J=13.5 \mathrm{~Hz}, 1 \mathrm{H}, \mathrm{CH}_{2} \mathrm{Ph}\right), 3.68\left(\mathrm{dd},{ }^{2} J=8.0 \mathrm{~Hz},{ }^{3} J=7.0 \mathrm{~Hz}\right.$, $1 \mathrm{H}, \mathrm{CH}_{2}-\mathrm{O}$ ), 2.74 (d, ${ }^{3} \mathrm{~J}=5.5 \mathrm{~Hz}, 2 \mathrm{H}, \mathrm{CH}_{2}-\mathrm{N}$ ), 1.64 (br s, $1 \mathrm{H}, \mathrm{NH}), 1.41(\mathrm{~s}, 3 \mathrm{H}, \mathrm{Me}), 1.35(\mathrm{~s}, 3 \mathrm{H}, \mathrm{Me}) ;{ }^{13} \mathrm{C}$ NMR $\left(100 \mathrm{MHz}, \mathrm{CDCl}_{3}\right): \delta 140.1(\mathrm{C}-\mathrm{Ar}), 128.3(\mathrm{C}-\mathrm{Ar}), 128.0$ (C-Ar), 126.9 (C-Ar), $109.0\left(\mathrm{C}-\left(\mathrm{CH}_{3}\right)_{2}\right), 75.4(\mathrm{CH}-\mathrm{O})$, $67.5\left(\mathrm{CH}_{2}-\mathrm{O}\right), 53.9\left(\mathrm{CH}_{2} \mathrm{Ph}\right), 51.7\left(\mathrm{CH}_{2} \mathrm{~N}\right), 26.8\left(\mathrm{CH}_{3}\right)$, $25.4\left(\mathrm{CH}_{3}\right)$; MS (ESI) $\mathrm{m} / z: 244[\mathrm{M}+\mathrm{Na}]^{+}, 222[\mathrm{M}+\mathrm{H}]^{+}$, $164[\mathrm{M}-\text { acetone }+\mathrm{H}]^{+}$; HRMS (ESI) calcd for $\mathrm{C}_{13} \mathrm{H}_{20} \mathrm{NO}_{2}$ $[\mathrm{M}+\mathrm{H}]^{+}: 222.1494$, found: 222.1508 .

4.2.4. 1,3-Bis\{benzy $[(( \pm)-2,2$-dimethyl-1,3-dioxolan4-yl)methyl]amino\}propanone $\boldsymbol{O}$-benzyloxime (8). To a stirred solution of amine $7(2.00 \mathrm{~g}, 8.8 \mathrm{mmol})$ in methanol $(10 \mathrm{~mL})$ was added a solution of oxime $4(0.49 \mathrm{~g}, 2.1 \mathrm{mmol})$ in methanol $(4 \mathrm{~mL})$. The resulting mixture was heated at reflux for 3 days. After evaporating to dryness, a saturated solution of $\mathrm{NaHCO}_{3}(40 \mathrm{~mL})$ was added followed by $\mathrm{CH}_{2} \mathrm{Cl}_{2}(100 \mathrm{~mL})$. The organic layer was dried over $\mathrm{MgSO}_{4}$ and concentrated. The crude mixture was purified by flash column chromatography using cyclohexane/ethyl acetate $(1: 0-4: 1,(\mathrm{v} / \mathrm{v}))$ as eluent to give 7 as an inseparable mixture of $(Z)$ - and $(E)$-isomers $(0.97 \mathrm{~g}, 77 \%) . R_{f}: 0.44$ (cyclohexane/ethyl acetate $=4: 1$ ); IR (film): $\nu$ 1250-1060 (C-O) $\mathrm{cm}^{-1}$; ${ }^{1} \mathrm{H}$ NMR (400 MHz, $\mathrm{CDCl}_{3}$ ): $\delta 7.38-7.18$ (m, 15H, H-Ar), $5.08\left(\mathrm{~s}, 2 \mathrm{H}, \mathrm{OCH}_{2} \mathrm{Ph}\right), 4.23-4.13(\mathrm{~m}, 2 \mathrm{H}$, $\left.\mathrm{H}-2,2^{\prime}\right), 3.91-3.86\left(\mathrm{~m}, 2 \mathrm{H}, \mathrm{H}-3,3^{\prime}\right), 3.71-3.35(\mathrm{~m}, 9 \mathrm{H}$, $\left.\mathrm{H}-3,3^{\prime}, \mathrm{a}, \mathrm{c}, \mathrm{NCH}_{2} \mathrm{Ph}\right), 3.25$ and 3.17 (d, ${ }^{2} J=13.0 \mathrm{~Hz}, 1 \mathrm{H}$, $\mathrm{H}-\mathrm{a}), 2.65-2.44\left(\mathrm{~m}, 4 \mathrm{H}, \mathrm{H}-1,1^{\prime}\right), 1.34-1.33-1.32-1.31$ (s, $12 \mathrm{H}, \mathrm{Me}) ;{ }^{13} \mathrm{C}$ NMR $\left(100 \mathrm{MHz}, \mathrm{CDCl}_{3}\right): \delta 157.5$ (C-b), 138.9 (C-Ar), 137.9 (C-Ar), 129.0 (C-Ar), 128.9 (C-Ar), 128.3 (C-Ar), 128.2 (C-Ar), 128.1 (C-Ar), 128.0 (C-Ar), 
127.7 (C-Ar), $127.0(\mathrm{C}-\mathrm{Ar}), 126.9(\mathrm{C}-\mathrm{Ar}), 109.0\left(\mathrm{C}-\left(\mathrm{CH}_{3}\right)_{2}\right)$, $75.8\left(\mathrm{OCH}_{2} \mathrm{Ph}\right), 74.5-74.2\left(\mathrm{C}-2,2^{\prime}\right), 68.4-68.35-68.3(\mathrm{C}-$ $\left.3,3^{\prime}\right), 59.9-58.7-58.6\left(\mathrm{NCH}_{2} \mathrm{Ph}\right), 57.2-57.1-56.4-56.3(\mathrm{C}-$ $\left.1,1^{\prime}\right), 55.4$ (C-a), 48.7 (C-c), 26.9-26.8 $\left(\mathrm{CH}_{3}\right), 25.7-25.6$ $\left(\mathrm{CH}_{3}\right) ; \mathrm{MS}$ (ESI) $m / z: 640[\mathrm{M}+\mathrm{K}]^{+}, 624[\mathrm{M}+\mathrm{Na}]^{+}$, $602[\mathrm{M}+\mathrm{H}]^{+}$; HRMS (ESI) calcd for $\mathrm{C}_{36} \mathrm{H}_{48} \mathrm{~N}_{3} \mathrm{O}_{5}[\mathrm{M}+\mathrm{H}]^{+}$: 602.3594, found: 602.3580 .

4.2.5. $N$-[(( \pm$)$-2,2-Dimethyl-1,3-dioxolan-4-yl)methyl]benzamide (9a). To a solution of amine 6 (300 mg, $2.3 \mathrm{mmol})$ in anhydrous $\mathrm{CH}_{2} \mathrm{Cl}_{2}(10 \mathrm{~mL})$ was successively added, at $0{ }^{\circ} \mathrm{C}$ and under argon, triethylamine $(480 \mu \mathrm{L}$, $3.4 \mathrm{mmol}$ ) and 4-dimethylaminopyridine ( $56 \mathrm{mg}, 0.5 \mathrm{mmol})$. After stirring for $15 \mathrm{~min}$, benzoyl chloride $(425 \mu \mathrm{L}$, $4.2 \mathrm{mmol}$ ) was added dropwise. The reaction mixture was stirred until the reaction was completed. The reaction was quenched with water $(4 \mathrm{~mL})$ and the reaction mixture was extracted with $\mathrm{CH}_{2} \mathrm{Cl}_{2}(3 \times 10 \mathrm{~mL})$. The organic layer was dried over $\mathrm{MgSO}_{4}$ and the solvent was removed in vacuo. The crude residue was purified by flash column chromatography using cyclohexane/ethyl acetate $(7: 3-3: 2$, $\mathrm{v} / \mathrm{v})$ as eluent and gave 9a as a white solid (405 mg, 75\%). $R_{f}: 0.20$ (cyclohexane/ethyl acetate $=7: 3$ ); mp $105^{\circ} \mathrm{C}$ (ethyl acetate); ${ }^{1} \mathrm{H}$ NMR $\left(400 \mathrm{MHz}, \mathrm{CDCl}_{3}\right): \delta 7.78(\mathrm{~m}, 2 \mathrm{H}, \mathrm{H}-$ Ar), 7.50 (m, 1H, H-Ar), 7.43 (m, 2H, H-Ar), 6.53 (br s, $1 \mathrm{H}, \mathrm{NH}), 4.34$ (qd, ${ }^{3} J=6.5$ and $\left.3.5 \mathrm{~Hz}, 1 \mathrm{H}, \mathrm{CH}-\mathrm{O}\right), 4.08$ (dd, ${ }^{2} J=8.5 \mathrm{~Hz},{ }^{3} J=6.5 \mathrm{~Hz}, 1 \mathrm{H}, \mathrm{CH}_{2}-\mathrm{O}$ ), 3.75 (ddd, ${ }^{2} J=14.0 \mathrm{~Hz},{ }^{3} \mathrm{~J}=6.0$ and $3.5 \mathrm{~Hz}, 1 \mathrm{H}, \mathrm{CH}_{2}-\mathrm{N}$ ), 3.71 (dd, $\left.{ }^{2} J=8.5 \mathrm{~Hz},{ }^{3} J=6.5 \mathrm{~Hz}, 1 \mathrm{H}, \mathrm{CH}_{2}-\mathrm{O}\right), 3.51\left(\mathrm{dt},{ }^{2} J=14.0 \mathrm{~Hz}\right.$, $\left.{ }^{3} J=6.0 \mathrm{~Hz}, 1 \mathrm{H}, \mathrm{CH}_{2}-\mathrm{N}\right), 1.45(\mathrm{~s}, 3 \mathrm{H}, \mathrm{Me}), 1.36(\mathrm{~s}, 3 \mathrm{H}$, $\mathrm{Me}) ;{ }^{13} \mathrm{C}$ NMR $\left(100 \mathrm{MHz}, \mathrm{CDCl}_{3}\right): \delta 167.7(\mathrm{C}=\mathrm{O}), 134.2$ (C-Ar), $131.6(\mathrm{C}-\mathrm{Ar}), 128.6(\mathrm{C}-\mathrm{Ar}), 126.9(\mathrm{C}-\mathrm{Ar}), 109.4$ $\left(\mathrm{C}-\left(\mathrm{CH}_{3}\right)_{2}\right), 74.6(\mathrm{CH}-\mathrm{O}), 66.7\left(\mathrm{CH}_{2}-\mathrm{O}\right), 41.9\left(\mathrm{CH}_{2}-\mathrm{N}\right)$, $26.8\left(\mathrm{CH}_{3}\right), 25.1\left(\mathrm{CH}_{3}\right)$.

4.2.6. $N-[(( \pm)-2,2-D i m e t h y l-1,3-d i o x o l a n-4-y l) m e t h y l]-$ 2,2,2-trifluoroacetamide (9b). A solution of amine 6 $(500 \mathrm{mg}, 4.81 \mathrm{mmol})$ and ethyl trifluoroacetate $(5.70 \mathrm{~mL}$, $48.1 \mathrm{mmol}$ ) was stirred at $20^{\circ} \mathrm{C}$ for $20 \mathrm{~h}$. After concentration, toluene was added and the resulting solution was evaporated under vacuo to eliminate all traces of solvent and reagent. Pure compound $9 \mathrm{~b}$ was obtained as a chestnut liquid (830 mg, 76\%). ${ }^{1} \mathrm{H}$ NMR (400 MHz, $\mathrm{CDCl}_{3}: \delta 6.77$ (br s, $1 \mathrm{H}$, $\mathrm{NH}), 4.27$ (qd, ${ }^{3} J=6.0$ and $3.5 \mathrm{~Hz}, 1 \mathrm{H}, \mathrm{CH}-\mathrm{O}$ ), 4.07 (dd, $\left.{ }^{2} J=8.5 \mathrm{~Hz},{ }^{3} J=6.5 \mathrm{~Hz}, 1 \mathrm{H}, \mathrm{CH}_{2}-\mathrm{O}\right), 3.65\left(\mathrm{dd},{ }^{2} J=8.5 \mathrm{~Hz}\right.$, ${ }^{3} J=6.0 \mathrm{~Hz}, 1 \mathrm{H}, \mathrm{CH}_{2}-\mathrm{O}$ ), 3.62 (ddd, ${ }^{2} J=14.0 \mathrm{~Hz},{ }^{3} J=6.0$ and $\left.3.5 \mathrm{~Hz}, 1 \mathrm{H}, \mathrm{CH}_{2}-\mathrm{N}\right), 3.37\left(\mathrm{dt},{ }^{2} J=14.0 \mathrm{~Hz},{ }^{3} J=6.0 \mathrm{~Hz}\right.$, $\left.1 \mathrm{H}, \mathrm{CH}_{2}-\mathrm{N}\right), 1.43(\mathrm{~s}, 3 \mathrm{H}, \mathrm{Me}), 1.34(\mathrm{~s}, 3 \mathrm{H}, \mathrm{Me}) ;{ }^{13} \mathrm{C}$ NMR $\left(100 \mathrm{MHz}, \mathrm{CDCl}_{3}\right): \delta 157.5\left(\mathrm{q},{ }^{2} J_{\mathrm{CF}}=37 \mathrm{~Hz}, \mathrm{C}=\mathrm{O}\right), 115.7$ $\left(\mathrm{q},{ }^{1} J_{\mathrm{CF}}=287 \mathrm{~Hz}, \mathrm{CF}_{3}\right), 109.9\left(\mathrm{C}-\left(\mathrm{CH}_{3}\right)_{2}\right), 73.4(\mathrm{CH}-\mathrm{O})$, $66.5\left(\mathrm{CH}_{2}-\mathrm{O}\right), 42.0\left(\mathrm{CH}_{2}-\mathrm{N}\right), 26.6\left(\mathrm{CH}_{3}\right), 24.9\left(\mathrm{CH}_{3}\right)$.

4.2.7. tert-Butyl[(( \pm )-2,2-dimethyl-1,3-dioxolan-4-yl)methyl]carbamate $(9 \mathrm{c})$. To a solution of $6(1.39 \mathrm{~g}$, $10.6 \mathrm{mmol})$ in dioxane $(85 \mathrm{~mL})$ was added, at $0^{\circ} \mathrm{C}$, a $0.5 \mathrm{M}$ aqueous solution of $\mathrm{Na}_{2} \mathrm{CO}_{3}(21.2 \mathrm{~mL}, 10.6 \mathrm{mmol})$ followed by di-tert-butyl dicarbonate $(2.5 \mathrm{~mL}, 11.7 \mathrm{mmol})$. The reaction mixture was stirred at $20^{\circ} \mathrm{C}$ for $14 \mathrm{~h}$ and then concentrated. The white precipitate thus obtained was dissolved in water and the aqueous layer was extracted with ethyl acetate. The combined organic layers were washed with brine and dried over $\mathrm{MgSO}_{4}$. The residue was purified by flash column chromatography using cyclohexane/ethyl acetate $(7: 3-3: 2, \mathrm{v} / \mathrm{v})$ as eluent and gave $9 \mathrm{c}$ as an oil $(2.04 \mathrm{~g}, 83 \%) . R_{f}: 0.43$ (cyclohexane/ethyl acetate $=7: 3$ ); IR (film): $\nu$ 3363, 1712, 1250-1050 $\mathrm{cm}^{-1}$; ${ }^{1} \mathrm{H}$ NMR $\left(400 \mathrm{MHz}, \mathrm{CDCl}_{3}\right): \delta 4.87$ (br s, $\left.1 \mathrm{H}, \mathrm{NH}\right), 4.17$ $(\mathrm{m}, 1 \mathrm{H}, \mathrm{CH}-\mathrm{O}), 4.01$ (dd, ${ }^{2} J=8.0 \mathrm{~Hz},{ }^{3} J=6.5 \mathrm{~Hz}, 1 \mathrm{H}$, $\mathrm{CH}_{2}-\mathrm{O}$ ), $3.63\left(\mathrm{dd},{ }^{2} \mathrm{~J}=8.0 \mathrm{~Hz},{ }^{3} \mathrm{~J}=6.5 \mathrm{~Hz}, 1 \mathrm{H}, \mathrm{CH}_{2}-\mathrm{O}\right.$ ), $3.37\left(\mathrm{~m}, 1 \mathrm{H}, \mathrm{CH}_{2}-\mathrm{N}\right), 3.16\left(\mathrm{dt},{ }^{2} J=14.0 \mathrm{~Hz},{ }^{3} J=6.0 \mathrm{~Hz}\right.$, $\left.1 \mathrm{H}, \mathrm{CH}_{2}-\mathrm{N}\right), 1.42(\mathrm{~s}, 9 \mathrm{H}, t-\mathrm{Bu}), 1.40(\mathrm{~s}, 3 \mathrm{H}, \mathrm{Me}), 1.32(\mathrm{~s}$, $3 \mathrm{H}, \mathrm{Me}) ;{ }^{13} \mathrm{C} \mathrm{NMR}\left(100 \mathrm{MHz}, \mathrm{CDCl}_{3}\right): \delta 156.0(\mathrm{C}=\mathrm{O})$, $109.2\left(\mathrm{C}-\left(\mathrm{CH}_{3}\right)_{2}\right), 79.4\left(\mathrm{C}-\left(\mathrm{CH}_{3}\right)_{3}\right), 74.9(\mathrm{CH}-\mathrm{O}), 66.6$ $\left(\mathrm{CH}_{2}-\mathrm{O}\right), 42.7\left(\mathrm{CH}_{2}-\mathrm{N}\right), 28.3\left(\left(\mathrm{CH}_{3}\right)_{3}\right), 26.7\left(\mathrm{CH}_{3}\right), 25.2$ $\left(\mathrm{CH}_{3}\right)$; HRMS (ESI) calcd for $\mathrm{C}_{11} \mathrm{H}_{22} \mathrm{NO}_{4} \mathrm{Na}[\mathrm{M}+\mathrm{Na}]^{+}$: 254.1368, found: 254.1369 .

4.2.8. tert-Butyl $\{2-[$ (benzyloxy)imino $]$-3-chloropropyl $\}$ [(( \pm )-2,2-dimethyl-1,3-dioxolan-4-yl)methyl]carbamate (11). To a suspension of $\mathrm{KH}(160 \mathrm{mg}, 1.20 \mathrm{mmol})$ in $25-$ $35 \%$ mineral oil was added, under argon, anhydrous THF ( $2 \mathrm{~mL})$ followed by a solution of $9 \mathrm{c}(200 \mathrm{mg}, 0.86 \mathrm{mmol})$ in THF $(1 \mathrm{~mL})$. When the bubbling had ceased, a solution of 4 ( $90 \mathrm{mg}, 0.38 \mathrm{mmol})$ in THF $(1 \mathrm{~mL})$ was introduced. The reaction mixture was stirred at $20^{\circ} \mathrm{C}$ for $2 \mathrm{~h}$. Then water $(2 \mathrm{~mL})$ was slowly added and the solution was diluted with $\mathrm{CH}_{2} \mathrm{Cl}_{2}(15 \mathrm{~mL})$. The layers were separated and the aqueous one was extracted twice with $\mathrm{CH}_{2} \mathrm{Cl}_{2}(5 \mathrm{~mL})$. The organic layer was then dried over $\mathrm{MgSO}_{4}$ and the solvent was evaporated. The residue was purified by flash column chromatography using cyclohexane/ethyl acetate $(19: 1, \mathrm{v} / \mathrm{v})$ as eluent to give a mixture of $(Z)$ - and $(E)$-oximes 11 as an oil (54 mg, $33 \%$ ). $R_{f} \cdot 0.63$ (cyclohexane/ethyl acetate $=7: 3$ ); IR (film): $\nu$ $1697(\mathrm{C}=\mathrm{O}), 1250-1000(\mathrm{C}-\mathrm{O}) \mathrm{cm}^{-1} ;{ }^{1} \mathrm{H} \mathrm{NMR}(400 \mathrm{MHz}$, $\left.\mathrm{CDCl}_{3}\right): \delta 7.34(\mathrm{~m}, 5 \mathrm{H}, \mathrm{H}-\mathrm{Ar}), 5.13\left(\mathrm{~s}, 2 \mathrm{H}, \mathrm{CH}_{2} \mathrm{Ph}\right), 4.48-$ $4.10\left(\mathrm{~m}, 5 \mathrm{H}, \mathrm{CH}_{2} \mathrm{Cl}, \mathrm{CH}_{2}-\mathrm{C}=\mathrm{N}\right.$ and $\left.\mathrm{CH}-\mathrm{O}\right), 4.00(\mathrm{~m}, 1 \mathrm{H}$, $\left.\mathrm{CH}_{2}-\mathrm{O}\right), 3.64-3.44\left(\mathrm{~m}, 2 \mathrm{H}, \mathrm{CH}_{2}-\mathrm{O}\right.$ and $\left.\mathrm{CH}_{2}-\mathrm{N}\right), 3.22(\mathrm{~m}$, $\left.1 \mathrm{H}, \mathrm{CH}_{2}-\mathrm{N}\right), 1.46$ and $1.42(\mathrm{~s}, 9 \mathrm{H}, t-\mathrm{Bu}), 1.38(\mathrm{~s}, 3 \mathrm{H}$, $\mathrm{Me}), 1.32(\mathrm{~s}, 3 \mathrm{H}, \mathrm{Me}) ;{ }^{13} \mathrm{C} \mathrm{NMR}\left(100 \mathrm{MHz}, \mathrm{CDCl}_{3}\right)$ : $\delta$ 155.7-155.6-155.3 $(\mathrm{C}=\mathrm{O}), \quad 154.5(\mathrm{C}=\mathrm{N}), \quad 137.1-$ 137.0-128.4-128.0 (C-Ar), $109.3\left(\mathrm{C}-\left(\mathrm{CH}_{3}\right)_{2}\right), 80.8-80.6$ $\left(\mathrm{C}-\left(\mathrm{CH}_{3}\right)_{3}\right), 76.7-76.4\left(\mathrm{CH}_{2} \mathrm{Ph}\right), 75.0-74.9(\mathrm{CH}-\mathrm{O}), 67.0$ $\left(\mathrm{CH}_{2}-\mathrm{O}\right), 51.1-50.8\left(\mathrm{CH}_{2}-\mathrm{N}\right), 44.1-43.8 \quad\left(\mathrm{CH}_{2}-\mathrm{CN}\right)$, $42.7-42.0\left(\mathrm{CH}_{2} \mathrm{Cl}\right), 28.2\left(\left(\mathrm{CH}_{3}\right)_{3}\right), 26.7\left(\mathrm{CH}_{3}\right), 25.5-25.4$ $\left(\mathrm{CH}_{3}\right)$; HRMS (ESI) calcd for $\mathrm{C}_{21} \mathrm{H}_{31} \mathrm{ClN}_{2} \mathrm{O}_{5} \mathrm{Na}[\mathrm{M}+\mathrm{Na}]^{+}$: 449.1819; found: 449.1834 .

4.2.9. 1,3-Diamino-propan-2-one $\boldsymbol{O}$-benzyloxime (12). To a solution of $4(1.83 \mathrm{~g}, 7.9 \mathrm{mmol})$ in dry DMF $(8 \mathrm{~mL})$ was added potassium phthalimide salt $(5.88 \mathrm{~g}, 31.8 \mathrm{mmol})$. The reaction mixture was heated to $100{ }^{\circ} \mathrm{C}$ for $3-4 \mathrm{~h}$. After cooling, the solution was diluted with water $(50 \mathrm{~mL})$ and extracted three times with $\mathrm{CH}_{2} \mathrm{Cl}_{2}(30 \mathrm{~mL})$. The combined organic layers were washed with brine, dried over $\mathrm{MgSO}_{4}$ and the solvent was evaporated to dryness. The residue was stored in a fridge until a precipitate appeared. After filtration, 2,2'-\{2-[(benzyloxy)imino]propane-1,3-diyl $\}$ bis[1 $\mathrm{H}$-isoindole-1,3(2H)-dione] intermediate was obtained as a white solid $(2.84 \mathrm{~g}, 84 \%)$. Mp $178^{\circ} \mathrm{C}$ (ethyl acetate); IR $(\mathrm{KBr}): \nu 1716(\mathrm{C}=\mathrm{O}) \mathrm{cm}^{-1} ;{ }^{1} \mathrm{H} \mathrm{NMR}\left(400 \mathrm{MHz}^{\mathrm{CDCl}} 3\right)$ : $\delta 7.81$ (dd, ${ }^{3} J=5.0 \mathrm{~Hz},{ }^{4} J=3.0 \mathrm{~Hz}, 2 \mathrm{H}, \mathrm{H}-\mathrm{Ar}$ ), 7.77 (dd, $\left.{ }^{3} J=5.0 \mathrm{~Hz},{ }^{4} J=3.0 \mathrm{~Hz}, 2 \mathrm{H}, \mathrm{H}-\mathrm{Ar}\right), 7.72\left(\mathrm{dd},{ }^{3} J=5.0 \mathrm{~Hz}\right.$, $\left.{ }^{4} J=3.0 \mathrm{~Hz}, 2 \mathrm{H}, \mathrm{H}-\mathrm{Ar}\right), 7.70\left(\mathrm{dd},{ }^{3} J=5.0 \mathrm{~Hz},{ }^{4} J=3.0 \mathrm{~Hz}\right.$, $2 \mathrm{H}, \mathrm{H}-\mathrm{Ar}), 7.21-7.17$ (m, 5H, H-Ar), 5.04 (s, 2H, $\left.\mathrm{CH}_{2} \mathrm{Ph}\right), 4.59\left(\mathrm{~s}, 2 \mathrm{H}, \mathrm{CH}_{2}-\mathrm{C}=\mathrm{N}\right), 4.84\left(\mathrm{~s}, 2 \mathrm{H}, \mathrm{CH}_{2}-\right.$ $\mathrm{C}=\mathrm{N}) ;{ }^{13} \mathrm{C}$ NMR $\left(100 \mathrm{MHz}, \mathrm{CDCl}_{3}\right): \delta \quad 167.5-167.4$ 
$(\mathrm{C}=\mathrm{O}), 148.8(\mathrm{C}=\mathrm{N}), 136.8(\mathrm{C}-\mathrm{Ar}), 133.9(\mathrm{C}-\mathrm{Ar}), 131.9$ (C-Ar), 131.8 (C-Ar), $128.1(\mathrm{C}-\mathrm{Ar}), 127.7$ (C-Ar), 123.3 $(\mathrm{C}-\mathrm{Ar}), 76.8\left(\mathrm{CH}_{2} \mathrm{Ph}\right), 39.0\left(\mathrm{CH}_{2}-\mathrm{C}=\mathrm{N}\right), 34.4\left(\mathrm{CH}_{2}-\right.$ $\mathrm{C}=\mathrm{N}$ ). Anal. Calcd for $\mathrm{C}_{26} \mathrm{H}_{19} \mathrm{~N}_{3} \mathrm{O}_{5}$ (453.45): $\mathrm{C}$, 68.87; H, 4.22; N, 9.27. Found: C, 68.74; H, 4.19; N, 9.29.

An aliquot of this intermediate $(500 \mathrm{mg}, 1.10 \mathrm{mmol})$ was dissolved in methanol $(10 \mathrm{~mL})$ and hydrazine hydrate $(160 \mu \mathrm{L}, 3.30 \mathrm{mmol})$ was added. The reaction mixture was boiled for $3 \mathrm{~h}$ and a solution of $\mathrm{KOH}(186 \mathrm{mg}, 3.30 \mathrm{mmol}$ ) in methanol $(5 \mathrm{~mL})$ was added. The resulting solution was stirred at $20^{\circ} \mathrm{C}$ overnight and the solvent and hydrazine were evaporated. Then, $\mathrm{CH}_{2} \mathrm{Cl}_{2}$ was added to the residue followed by $\mathrm{MgSO}_{4}$. After filtration, the crude amine 12 was not isolated but kept as a solution in anhydrous $\mathrm{CH}_{2} \mathrm{Cl}_{2}$ under argon. ${ }^{1} \mathrm{H}$ NMR $\left(400 \mathrm{MHz}, \mathrm{CDCl}_{3}\right): \delta 7.37-7.30(\mathrm{~m}, 5 \mathrm{H}$, $\mathrm{H}-\mathrm{Ar}), 5.09$ (s, 2H, $\left.\mathrm{CH}_{2} \mathrm{Ph}\right), 3.57\left(\mathrm{~s}, 2 \mathrm{H}, \mathrm{CH}_{2}-\mathrm{N}\right), 3.49$ (s, $2 \mathrm{H}, \mathrm{CH}_{2}-\mathrm{N}$ ), 1.45 (br s, $4 \mathrm{H}, \mathrm{NH}_{2}$ ).

4.2.10. $N, N^{\prime}-\{2-[($ Benzyloxy)imino]propane-1,3-diyl $\}$-bisbenzamide (13a). To a stirred and ice-cooled solution of 1,3diamino-propan-2-one $O$-benzyloxime dihydrochloride 12 (1.60 mmol) in $\mathrm{CH}_{2} \mathrm{Cl}_{2}$ and under argon were added triethylamine $(670 \mu \mathrm{L}, 4.80 \mathrm{mmol})$ and 4-dimethylaminopyridine $(0.64 \mathrm{mmol})$ followed by dropwise addition of a solution of benzoyl chloride $(600 \mu \mathrm{L}, 5.12 \mathrm{mmol})$. The reaction mixture was stirred for $3 \mathrm{~h}$ at $20^{\circ} \mathrm{C}$ and $\mathrm{CH}_{2} \mathrm{Cl}_{2}(60 \mathrm{~mL})$ was added. The organic layer was washed twice with water $(12 \mathrm{~mL})$ following by a saturated $\mathrm{NaHCO}_{3}$ solution $(12 \mathrm{~mL})$, dried over $\mathrm{MgSO}_{4}$ and concentrated in vacuo. The residue was purified by flash column chromatography using ethyl acetate/cyclohexane $(4: 6-1: 1, \mathrm{v} / \mathrm{v})$ as eluent to give 13a (435 mg, 68\%) as a white solid. Mp $136{ }^{\circ} \mathrm{C} ; R_{f}: 0.49$ (ethyl acetate/cyclohexane $=1: 1) ;{ }^{1} \mathrm{H}$ NMR $\left(400 \mathrm{MHz}, \mathrm{CDCl}_{3}\right)$ : $\delta 7.88\left(\mathrm{~d},{ }^{3} \mathrm{~J}=7.5 \mathrm{~Hz}, 2 \mathrm{H}, \mathrm{H}-\mathrm{Ar}\right), 7.78\left(\mathrm{~d},{ }^{3} \mathrm{~J}=7.5 \mathrm{~Hz}, 2 \mathrm{H}\right.$, $\mathrm{H}-\mathrm{Ar}$ ), 7.56 (br t, $\left.{ }^{3} \mathrm{~J}=7.0 \mathrm{~Hz}, 1 \mathrm{H}, \mathrm{NH}\right), 7.51$ (t, ${ }^{3} J=7.5 \mathrm{~Hz}$, $2 \mathrm{H}, \mathrm{H}-\mathrm{Ar}), 7.44\left(\mathrm{t},{ }^{3} \mathrm{~J}=7.5 \mathrm{~Hz}, 2 \mathrm{H}, \mathrm{H}-\mathrm{Ar}\right), 7.42$ (t, $\left.{ }^{3} J=7.5 \mathrm{~Hz}, 2 \mathrm{H}, \mathrm{H}-\mathrm{Ar}\right), 7.33(\mathrm{M}, 6 \mathrm{H}, \mathrm{H}-\mathrm{Bn}$ and $\mathrm{NH}), 5.14$ (s, $\left.2 \mathrm{H}, \mathrm{CH}_{2} \mathrm{Ph}\right), 4.36\left(\mathrm{~d},{ }^{3} \mathrm{~J}=6.5 \mathrm{~Hz}, 2 \mathrm{H}, \mathrm{H}-\mathrm{a}\right), 4.27$ (d, $\left.{ }^{3} J=5.0 \mathrm{~Hz}, 2 \mathrm{H}, \mathrm{H}-\mathrm{c}\right)$. Anal. Calcd for $\mathrm{C}_{24} \mathrm{H}_{23} \mathrm{~N}_{3} \mathrm{O}_{3}$ (401.46): C, 71.80; H, 5.77; N, 10.47. Found: C, 72.27; H, $5.81 ; \mathrm{N}, 10.44$.

4.2.11. tert-Butyl\{2-[(benzyloxy)imino]propane-1,3diyl\}biscarbamate (13b). To a stirred and ice-cooled solution of crude $12(0.48 \mathrm{mmol})$ in dioxane $(4 \mathrm{~mL})$, was added an aqueous $\mathrm{Na}_{2} \mathrm{CO}_{3}$ solution $(0.5 \mathrm{M}$ aq, $2.0 \mathrm{~mL}, 0.96 \mathrm{mmol})$ followed by di-tert-butyl dicarbonate $(230 \mu \mathrm{L}, 1.06 \mathrm{mmol})$. The reaction mixture was stirred at $20^{\circ} \mathrm{C}$ overnight. After removing the solvent, the residue was dissolved in water. The aqueous layer was extracted with ethyl acetate. The organic layer was washed with brine, dried over $\mathrm{MgSO}_{4}$ and concentrated in vacuo. The residue was purified by flash column chromatography using ethyl acetate/cyclohexane (2:8, $\mathrm{v} / \mathrm{v})$ as eluent to give $\mathbf{1 3 b}$ as a white solid (143 mg, 76\%). Mp $112^{\circ} \mathrm{C}$ (cyclohexane); ${ }^{1} \mathrm{H}$ NMR $\left(400 \mathrm{MHz}, \mathrm{CDCl}_{3}\right)$ : $\delta$ 7.37-7.31 (M, 5H, H-Ar), $5.19(\mathrm{se}, 1 \mathrm{H}, \mathrm{NH}), 5.10(\mathrm{~s}$, $\left.2 \mathrm{H}, \mathrm{CH}_{2} \mathrm{Ph}\right), 5.07$ (se, $\left.1 \mathrm{H}, \mathrm{NH}\right), 4.02\left(\mathrm{de},{ }^{3} \mathrm{~J}=5.0 \mathrm{~Hz}, 2 \mathrm{H}\right.$, $\mathrm{H}-\mathrm{c}), 3.92$ (dd, $\left.{ }^{3} \mathrm{~J}=5.0 \mathrm{~Hz}, 2 \mathrm{H}, \mathrm{H}-\mathrm{a}\right), 1.45(\mathrm{~s}, 9 \mathrm{H}, t-\mathrm{Bu})$, $1.44(\mathrm{~s}, 9 \mathrm{H}, t-\mathrm{Bu}) ;{ }^{13} \mathrm{C}$ NMR $\left(100 \mathrm{MHz}, \mathrm{CDCl}_{3}\right): \delta 156.0$ (CO), 155.7 (C-c), 154.8 (CO), 137.4 (C-Ar), $128.4(\mathrm{CH}-$ $\mathrm{Ar}), 128.0(\mathrm{CH}-\mathrm{Ar}), 127.9(\mathrm{CH}-\mathrm{Ar}), 79.9\left(\mathrm{C}-\left(\mathrm{CH}_{3}\right)_{3}\right)$, $79.6\left(\mathrm{C}-\left(\mathrm{CH}_{3}\right)_{3}\right), 76.3\left(\mathrm{CH}_{2} \mathrm{Ph}\right), 41.6(\mathrm{C}-\mathrm{c}), 37.0(\mathrm{C}-\mathrm{a})$,
$28.3\left(\mathrm{CH}_{3}\right)$. Anal. Calcd for $\mathrm{C}_{20} \mathrm{H}_{31} \mathrm{~N}_{3} \mathrm{O}_{5}$ (393.48): C, $61.05 ; \mathrm{H}, 7.94 ; \mathrm{N} 10.68$. Found: C, 61.22; H, 8.03; N, 10.74 .

4.2.12. (+)- $N, N^{\prime}-\{2-[($ Benzyloxy $)$ imino $]$ propane-1,3diyl $\}$ bis $\{[(4 R)$-2,2-dimethyl-1,3-dioxolan-4-yl]carboxamide (16). To a solution of crude amine 12 (16.2 mmol) in $\mathrm{CH}_{2} \mathrm{Cl}_{2}(170 \mathrm{~mL})$ were added, at $0{ }^{\circ} \mathrm{C}$ and under argon, triethylamine $(6.8 \mathrm{~mL}, 48.6 \mathrm{mmol})$ and 4-dimethylaminopyridine $(0.8 \mathrm{~g}, 6.5 \mathrm{mmol})$. After about $15 \mathrm{~min}$, acylchloride $(R)-15(5.8 \mathrm{~g}, 35.6 \mathrm{mmol})$ was slowly added and the stirring was continued until the reaction was completed (monitored by TLC). The reaction was quenched by $\mathrm{H}_{2} \mathrm{O}$ and the resulting solution was extracted with $\mathrm{CH}_{2} \mathrm{Cl}_{2}(3 \times 50 \mathrm{~mL})$. The combined organic layers were dried over $\mathrm{MgSO}_{4}$ and the solvent was evaporated. The residue was subjected to flash column chromatography using ethyl acetate/cyclohexane $(3: 2, v / v)$ as eluent to give compound 16 as an oil $(4.9 \mathrm{~g}$, $67 \%) .[\alpha]_{\mathrm{D}}^{25}+1.9\left(c 1.1, \mathrm{CHCl}_{3}\right)$; IR (film): $\nu$ 3413, 3337, $1736, \quad 1682, \quad 1300-1000 \mathrm{~cm}^{-1} ;{ }^{1} \mathrm{H}$ NMR $(400 \mathrm{MHz}$, $\left.\mathrm{CDCl}_{3}\right): \delta 7.37-7.31(\mathrm{M}, 5 \mathrm{H}, \mathrm{H}-\mathrm{Ar}), 7.26\left(\mathrm{se}, 1 \mathrm{H}, \mathrm{NH}_{\mathrm{a}}\right)$, $7.20\left(\mathrm{t}, J=6.0 \mathrm{~Hz}, 1 \mathrm{H}, \mathrm{NH}_{\mathrm{c}}\right), 5.10\left(\mathrm{~s}, 2 \mathrm{H}, \mathrm{CH}_{2} \mathrm{Ph}\right), 4.48$ (dd, ${ }^{3} J=8.5$ and $\left.4.0 \mathrm{~Hz}, 1 \mathrm{H}, \mathrm{H}-2^{\prime}\right), 4.47$ (dd, ${ }^{3} J=8.5$ and $4.0 \mathrm{~Hz}, 1 \mathrm{H}, \mathrm{H}-2), 4.26\left(\mathrm{t},{ }^{2} J=8.5 \mathrm{~Hz},{ }^{3} J=8.5 \mathrm{~Hz}, 2 \mathrm{H}, \mathrm{H}-\right.$ $\left.3,3^{\prime}\right), 4.19\left(\mathrm{dd},{ }^{2} J=16.0 \mathrm{~Hz},{ }^{3} J=6.5 \mathrm{~Hz}, 1 \mathrm{H}, \mathrm{H}-\mathrm{c}\right), 4.12$ (dd, $\left.{ }^{2} J=16.0 \mathrm{~Hz},{ }^{3} J=6.5 \mathrm{~Hz}, 1 \mathrm{H}, \mathrm{H}-\mathrm{a}\right), 4.11-4.05$ (M, 3H, $\mathrm{H}-\mathrm{c}, 3,3^{\prime}$ ), 4.01 (dd, $\left.{ }^{2} J=17.0 \mathrm{~Hz},{ }^{3} J=5.0 \mathrm{~Hz}, 1 \mathrm{H}, \mathrm{H}-\mathrm{a}\right), 1.46$ $(\mathrm{s}, 3 \mathrm{H}, \mathrm{Me}), 1.40(\mathrm{~s}, 3 \mathrm{H}, \mathrm{Me}), 1.38(\mathrm{~s}, 3 \mathrm{H}, \mathrm{Me}), 1.36(\mathrm{~s}$, $3 \mathrm{H}, \mathrm{Me}) ;{ }^{13} \mathrm{C}$ NMR $\left(100 \mathrm{MHz}, \mathrm{CDCl}_{3}\right): \delta 171.6\left(\mathrm{C}-1^{\prime}\right)$, 171.4 (C-1), 152.8 (C-b), 137.0 (C-Ar), 128.4 (C-Ar), 128.1 (C-Ar), 128.0 (C-Ar), $111.0\left(\mathrm{C}-\left(\mathrm{CH}_{3}\right)_{2}\right), 76.6$ $\left(\mathrm{CH}_{2} \mathrm{Ph}\right), 75.0\left(\mathrm{C}-2^{\prime}\right), 74.9(\mathrm{C}-2), 67.7\left(\mathrm{C}-3^{\prime}\right), 67.6(\mathrm{C}-3)$, 40.1 (C-a), 35.4 (C-c), $26.1\left(\mathrm{CH}_{3}\right), 26.0\left(\mathrm{CH}_{3}\right), 25.0$ $\left(\mathrm{CH}_{3}\right), 24.9\left(\mathrm{CH}_{3}\right) ; \mathrm{MS} \mathrm{m} / z: 472[\mathrm{M}+\mathrm{Na}]^{+}, 450[\mathrm{M}+\mathrm{H}]^{+}$. Anal. Calcd for $\mathrm{C}_{22} \mathrm{H}_{31} \mathrm{~N}_{3} \mathrm{O}_{7}$ : C, 58.78; $\mathrm{H}, 6.95 ; \mathrm{N}, 9.35$. Found: C, 58.62; H, 7.22; N, 9.25.

4.2.13. $N, N^{\prime}$-(2-Oxopropane-1,3-diyl)bis [(2R)-2,3-dihydroxypropanamide] (17). To a solution of $16(500 \mathrm{mg}$, $1.11 \mathrm{mmol})$ in acetone/water $(10: 1, \mathrm{v} / \mathrm{v})$, was added Amberlyst ${ }^{\circledR} 15(280 \mathrm{mg})$. The resulting suspension was heated under reflux for $15 \mathrm{~h}$. After cooling and filtration, a solid was obtained and recrystallized in ethanol to give pure 17 (226 mg, 77\%). $R_{f} \cdot 0.05$ (ethyl acetate/methanol=4:1). The ${ }^{1} \mathrm{H}$ NMR (DMSO- $d_{6}$, at $20^{\circ} \mathrm{C}$ ) spectrum could not be attributed because of a coalescence phenomenon; ${ }^{13} \mathrm{C}$ NMR (100 MHz, DMSO- $d_{6}$ ): $\delta 203.0$ (CO), 172.4 (NHCO), 72.9 $(\mathrm{CHOH}), 63.8\left(\mathrm{CH}_{2} \mathrm{OH}\right), 46.4\left(\mathrm{CH}_{2} \mathrm{NH}\right)$; $\mathrm{MS}$ (ESI) $\mathrm{m} / \mathrm{z}$ : $303[\mathrm{M}+\mathrm{K}]^{+}, \quad 287 \quad[\mathrm{M}+\mathrm{Na}]^{+}, \quad 265 \quad[\mathrm{M}+\mathrm{H}]^{+}, \quad 247$ $\left[\mathrm{M}-\mathrm{H}_{2} \mathrm{O}+\mathrm{H}\right]^{+}$.

4.2.14. (-)-(2R)-2,3-Dihydroxy- $N-[(1 R, 5 R)$-(2-0xo-6,8dioxa-3-azabicyclo[3.2.1]oct-5-yl)methyl]propanamide (18). A stirred solution of 7 ( $465 \mathrm{mg}, 1.76 \mathrm{mmol}$ ) was refluxing in 1-butanol $(120 \mathrm{~mL})$ for $6 \mathrm{~h}$ with catalytic amounts of $p$ TsOH $(7 \mathrm{mg}, 0.03 \mathrm{mmol})$. The reaction mixture was then filtered through a Celite ${ }^{\circledR}$ pad and the solvent was eliminated. The residue was dissolved in $80 \mathrm{~mL}$ of water and washed three times with diethyl ether $(20 \mathrm{~mL})$. After evaporation of the water, the solid was recrystallized in ethanol to give 18 as a white powder (330 mg, 76\%).

(-)-( $2 R, 6 S, 8 R)-18: \mathrm{mp} 156^{\circ} \mathrm{C}$ (ethanol); $R_{f} 0.18$ (ethyl acetate/methanol $=4: 1) ;[\alpha]_{\mathrm{D}}^{25}-21.7\left(c 0.5, \mathrm{H}_{2} \mathrm{O}\right) ; \mathrm{IR}(\mathrm{KBr}) \nu$ 
3362, 3291, 1664, $1637 \mathrm{~cm}^{-1} ;{ }^{1} \mathrm{H} \quad \mathrm{NMR} \quad(400 \mathrm{MHz}$, $\left.\mathrm{CD}_{3} \mathrm{OD}\right): \delta 7.77(\mathrm{se}, 1 \mathrm{H}, \mathrm{NH}), 7.65\left(\mathrm{t},{ }^{3} J=6.0 \mathrm{~Hz}, 1 \mathrm{H}\right.$ $\mathrm{NH}), 5.59\left(\mathrm{~d},{ }^{3} J=5.5 \mathrm{~Hz}, 1 \mathrm{H}, \mathrm{OH}\right), 4.72\left(\mathrm{t},{ }^{3} J=5.5 \mathrm{~Hz}\right.$, $1 \mathrm{H}, \quad \mathrm{OH}), 4.64\left(\mathrm{~d},{ }^{3} J=5.0 \mathrm{~Hz}, 1 \mathrm{H}, \mathrm{H}-1^{\prime}\right), 4.02$ (d, $\left.{ }^{3} J=7.5 \mathrm{~Hz}, 1 \mathrm{H}, \mathrm{H}-7^{\prime} \mathrm{a}\right), 3.91\left(\mathrm{td},{ }^{3} J=5.5,5.5\right.$ and $3.5 \mathrm{~Hz}$, $1 \mathrm{H}, \mathrm{H}-2$ ), 3.79 (dd, ${ }^{2} J=7.5 \mathrm{~Hz},{ }^{3} J=5.0 \mathrm{~Hz}, 1 \mathrm{H}, \mathrm{H}-7^{\prime} \mathrm{b}$ ), 3.57 (ddd, ${ }^{2} J=11.0 \mathrm{~Hz},{ }^{3} J=5.5$ and $3.5 \mathrm{~Hz}, 1 \mathrm{H}, \mathrm{H}-3$ ), 3.51 (d, $\left.{ }^{3} J=6.0 \mathrm{~Hz}, 2 \mathrm{H}, \quad \mathrm{CH}_{2}-\mathrm{N}\right), 3.46 \quad\left(\mathrm{dt},{ }^{2} J=11.0 \mathrm{~Hz}\right.$, $\left.{ }^{3} J=5.5 \mathrm{~Hz}, 1 \mathrm{H}, \mathrm{H}-3\right), 3.33$ (m, $\left.1 \mathrm{H}, \mathrm{H}-4^{\prime} \mathrm{a}\right), 3.01$ (dd, $\left.{ }^{2} J=12.5 \mathrm{~Hz},{ }^{3} J=3.0 \mathrm{~Hz}, 1 \mathrm{H}, \mathrm{H}-4^{\prime} \mathrm{b}\right) ;{ }^{13} \mathrm{C}$ NMR $(100 \mathrm{MHz}$, DMSO- $\left.d_{6}\right): \delta 172.4(\mathrm{C}-1), 168.3\left(\mathrm{C}-2^{\prime}\right), 104.5\left(\mathrm{C}-5^{\prime}\right), 74.9$ $\left(\mathrm{C}-1^{\prime}\right), 72.8$ (C-2), $69.9\left(\mathrm{C}-7^{\prime}\right), 63.7(\mathrm{C}-3), 47.7$ (C-4'), $41.1\left(\mathrm{CH}_{2}-\mathrm{N}\right)$. Anal. Calcd for $\mathrm{C}_{9} \mathrm{H}_{14} \mathrm{~N}_{2} \mathrm{O}_{6}$ (246.2): C, 43.90; H, 5.73; N, 11.38. Found: C, 44.08; H, 5.83; N, 11.14; MS m/z: $269[\mathrm{M}+\mathrm{Na}]^{+}, 247[\mathrm{M}+\mathrm{H}]^{+}$.

4.2.15. $N, N^{\prime}-\{2-[($ Benzyloxy)imino $]$ propane-1,3-diyl $\}$ bis[(2R)-2,3-dihydroxypropanamide] (21). A solution of $16(1.00 \mathrm{~g}, 2.23 \mathrm{mmol})$ was heated at reflux in ethanol $(30 \mathrm{~mL})$ with catalytic amount of $\mathrm{D}, \mathrm{L}$-camphorsulfonic acid $(0.01 \mathrm{~g}, 0.04 \mathrm{mmol})$. After the reaction was completed, $\mathrm{K}_{2} \mathrm{CO}_{3}$ was added and the reaction mixture was filtered off. The solvent was evaporated to dryness to give $21(0.82 \mathrm{~g}$, quantitative yield). $R_{f}: 0.30$ (ethyl acetate/methanol, $4: 1$ ); ${ }^{1} \mathrm{H}$ NMR $\left(400 \mathrm{MHz}, \mathrm{CD}_{3} \mathrm{OD}\right): \delta 7.38-7.25(\mathrm{M}, 5 \mathrm{H}, \mathrm{H}-$ $\mathrm{Ar}), 5.09$ (s, 2H, $\left.\mathrm{CH}_{2} \mathrm{Ph}\right), 4.21$ (s, 2H, H-c), 4.13 (t, ${ }^{3} \mathrm{~J}=$ $\left.4.0 \mathrm{~Hz}, 1 \mathrm{H}, \mathrm{H}-2^{\prime}\right), 4.11\left(\mathrm{t},{ }^{3} J=4.0 \mathrm{~Hz}, 1 \mathrm{H}, \mathrm{H}-2\right), 4.01$ (d, $\left.{ }^{2} J=16.0 \mathrm{~Hz}, 1 \mathrm{H}, \mathrm{H}-\mathrm{a}\right), 3.98\left(\mathrm{~d},{ }^{2} J=16.0 \mathrm{~Hz}, 1 \mathrm{H}, \mathrm{H}-\mathrm{a}\right)$, $3.80-3.72$ (M, 4H, H-3, $\left.3^{\prime}\right) ;{ }^{13} \mathrm{C}$ NMR (100 MHz, $\left.\mathrm{CD}_{3} \mathrm{OD}\right)$ : $\delta 175.7\left(\mathrm{C}-1^{\prime}\right), 175.1$ (C-1), 155.5 (C-b), 139.0 (C-Ar), $129.4(\mathrm{C}-\mathrm{Ar}), 129.3$ (C-Ar), $128.9(\mathrm{C}-\mathrm{Ar}), 77.4\left(\mathrm{CH}_{2} \mathrm{Ph}\right)$, $74.3\left(\mathrm{C}-2,2^{\prime}\right), 65.3\left(\mathrm{C}-3^{\prime}\right), 65.2(\mathrm{C}-3), 40.7(\mathrm{C}-\mathrm{a}), 36.5(\mathrm{C}-\mathrm{c})$.

4.2.16. $N, N^{\prime}-\{2-[($ Benzyloxy $)$ imino $]$ propane-1,3-diyl $\}$ bis[(2R)-3-(benzyloxy)-2-hydroxypropanamide] (22a). A solution of $21(0.10 \mathrm{~g}, 0.27 \mathrm{mmol})$ in toluene/methanol $(5.5 \mathrm{~mL}, 10: 1, \mathrm{v} / \mathrm{v})$ was heated at reflux until complete dissolution. Then, di- $n$-butyltin oxide $(0.14 \mathrm{~g}, 0.57 \mathrm{mmol})$ was added and the resulting solution was heated using a Dean-Stark apparatus for 5-6 h. To the stirred resulting mixture were added benzylbromide $(0.13 \mathrm{~mL}, 1.10 \mathrm{mmol})$ and tetrabutylammonium iodide $(0.07 \mathrm{~g}, 0.19 \mathrm{mmol})$ and the heating was continued for $5 \mathrm{~h}$. After cooling, ethyl acetate $(15 \mathrm{~mL})$ and water $(5 \mathrm{~mL})$ were poured in the reaction mixture. The layers were separated and the aqueous one was further extracted with ethyl acetate $(3 \times 10 \mathrm{~mL})$, dried $\left(\mathrm{MgSO}_{4}\right)$ and the solvent evaporated until dryness. The residue was purified by flash column chromatography using ethyl acetate/cyclohexane $(19: 1-1: 0, \mathrm{v} / \mathrm{v})$ as eluent and gave 22a $(50 \mathrm{mg}, 34 \%) . R_{f}: 0.30$ (ethyl acetate); ${ }^{1} \mathrm{H}$ NMR $\left(400 \mathrm{MHz}, \mathrm{CDCl}_{3}\right): \delta 7.31-7.23(\mathrm{M}, 17 \mathrm{H}, \mathrm{H}-\mathrm{Ar}$ and $\mathrm{NH})$, $5.03\left(\mathrm{~s}, 2 \mathrm{H}, \mathrm{OCH}_{2} \mathrm{Ph}\right), 4.51\left(\mathrm{~d},{ }^{2} J=12.5 \mathrm{~Hz}, 2 \mathrm{H}, \mathrm{H}-\mathrm{Ar}\right)$, $4.49\left(\mathrm{~d},{ }^{2} J=12.5 \mathrm{~Hz}, 2 \mathrm{H}, \mathrm{H}-\mathrm{Ar}\right), 4.20\left(\mathrm{q},{ }^{3} \mathrm{~J}=5.0 \mathrm{~Hz}, 1 \mathrm{H}\right.$, $\mathrm{CHOH}), 4.19\left(\mathrm{q},{ }^{3} \mathrm{~J}=5.0 \mathrm{~Hz}, 1 \mathrm{H}, \mathrm{CHOH}\right), 4.13$ (dd, $\left.{ }^{2} J=16.5 \mathrm{~Hz},{ }^{3} J=6.5 \mathrm{~Hz}, 1 \mathrm{H}, \mathrm{H}-\mathrm{c}\right), 3.96\left(\mathrm{dd},{ }^{2} J=16.5 \mathrm{~Hz}\right.$, $\left.{ }^{3} J=6.5 \mathrm{~Hz}, 1 \mathrm{H}, \mathrm{H}-\mathrm{c}\right), 3.93$ (d, $\left.{ }^{3} J=6.0 \mathrm{~Hz}, 2 \mathrm{H}, \mathrm{H}-\mathrm{a}\right), 3.74$ (dd, ${ }^{2} J=9.5 \mathrm{~Hz},{ }^{3} J=5.0 \mathrm{~Hz}, 1 \mathrm{H}, \mathrm{H}-\mathrm{CH}_{2} \mathrm{O}$ ), 3.69 (d, ${ }^{3} J=$ $4.0 \mathrm{~Hz}, 2 \mathrm{H}, \mathrm{CH}_{2} \mathrm{O}$ ), 3.67 (dd, ${ }^{2} J=9.5 \mathrm{~Hz},{ }^{3} J=4.5 \mathrm{~Hz}, 1 \mathrm{H}$, $\left.\mathrm{CH}_{2} \mathrm{O}\right), 3.64\left(\mathrm{~d},{ }^{3} \mathrm{~J}=5.0 \mathrm{~Hz}, 1 \mathrm{H}, \mathrm{OH}\right), 3.57\left(\mathrm{~d},{ }^{3} \mathrm{~J}=5.0 \mathrm{~Hz}\right.$, $1 \mathrm{H}, \quad \mathrm{OH}) ;{ }^{13} \mathrm{C} \quad \mathrm{NMR} \quad\left(100 \mathrm{MHz}, \mathrm{CD}_{3} \mathrm{OD}\right): \delta 172.3$ (NHCO), 172.1 (NHCO), 154.0 (CO), 137.4 (C-Ar), 137.3 (C-Ar), 137.2 (C-Ar), 128.5 (C-Ar), 128.4 (C-Ar), 128.2 (C-Ar), 128.0 (C-Ar), 127.9 (C-Ar), 127.8 (C-Ar), 76.4
$\left(\mathrm{NOCH}_{2} \mathrm{Ph}\right), \quad 73.5\left(\mathrm{OCH}_{2} \mathrm{Ph}\right), \quad 73.4 \quad\left(\mathrm{OCH}_{2} \mathrm{Ph}\right), \quad 71.4$ $(\mathrm{CHOH}), 71.3\left(\mathrm{CH}_{2} \mathrm{O}\right), 39.9(\mathrm{C}-\mathrm{a}), 35.4(\mathrm{C}-\mathrm{c})$.

4.2.17. $N, N^{\prime}$-(2-Oxopropane-1,3-diyl)bis[(2R)-3-(benzy]oxy)-2-hydroxypropanamide] (23). To a solution of 22 (38 mg, $0.069 \mathrm{mmol})$ in acetone/water $(10 / 1, \mathrm{v} / \mathrm{v})$ was added Amberlyst ${ }^{\otimes} 15(20 \mathrm{mg})$. The reaction mixture was heated at reflux for $48 \mathrm{~h}$. After filtration on a Celite ${ }^{\otimes}$ pad, the residue was purified by flash column chromatography using ethyl acetate/methanol $(1: 0-24: 1, \mathrm{v} / \mathrm{v})$ as eluent to give 23 (26 mg, 85\%); $R_{f} 0.24$ (ethyl acetate); ${ }^{1} \mathrm{H} \mathrm{NMR} \mathrm{(400} \mathrm{MHz,}$ $\left.\mathrm{CDCl}_{3}\right): \delta 7.51(\mathrm{t}, J=5.0 \mathrm{~Hz}, 2 \mathrm{H}, \mathrm{NH}), 7.26-7.32(\mathrm{M}, 10 \mathrm{H}$, $\mathrm{H}-\mathrm{Ar}), 4.54\left(\mathrm{~s}, 4 \mathrm{H}, \mathrm{OCH}_{2} \mathrm{Ph}\right), 4.25(\mathrm{~m}, 2 \mathrm{H}, \mathrm{CHOH}), 4.14$ (dd, $\left.{ }^{2} J=18.0 \mathrm{~Hz},{ }^{3} J=6.0 \mathrm{~Hz}, 2 \mathrm{H}, \mathrm{H}-\mathrm{a}, \mathrm{c}\right), 4.06$ (dd, ${ }^{2} J=$ $\left.18.0 \mathrm{~Hz},{ }^{3} \mathrm{~J}=5.0 \mathrm{~Hz}, 2 \mathrm{H}, \mathrm{H}-\mathrm{a}, \mathrm{c}\right), 3.85\left(\mathrm{~m}, 2 \mathrm{H}, \mathrm{CH}_{2} \mathrm{O}\right), 3.74$ $\left(\mathrm{m}, 2 \mathrm{H}, \mathrm{CH}_{2} \mathrm{O}\right), 3.72\left(\mathrm{~d},{ }^{3} \mathrm{~J}_{32}=5.5 \mathrm{~Hz}, 2 \mathrm{H}, \mathrm{OH}\right) ;{ }^{13} \mathrm{C}$ NMR $\left(100 \mathrm{MHz}, \mathrm{CD}_{3} \mathrm{OD}\right): \delta 201.2$ (CO), 172.4 (NHCO), 137.3 (C-Ar), 128.5 (C-Ar), 128.0 (C-Ar), 127.9 (C-Ar), 73.5 $\left(\mathrm{OCH}_{2} \mathrm{Ph}\right), 71.2\left(\mathrm{CH}_{2} \mathrm{O}\right), 70.9(\mathrm{CHOH}), 46.9\left(\mathrm{CH}_{2} \mathrm{NH}\right)$.

4.2.18. (-)-Methyl-(2R)-3-(tert-butyldiphenylsilyloxy)2-hydroxypropanoate (25). To a solution of $(+)$-methyl$(2 R)$-2,3-dihydroxypropanoate $\mathbf{2 4}$ in anhydrous dichloromethane under argon was added imidazole $(0.57 \mathrm{mg}$, $8.3 \mathrm{mmol})$. The resulting mixture was cooled at $-40^{\circ} \mathrm{C}$ and tert-butyldiphenylchlorosilane $(1.37 \mathrm{~g}, 5.0 \mathrm{mmol})$ was added. The stirring was pursued for $1 \mathrm{~h} 30 \mathrm{~min}$ and then the reaction was quenched by a saturated solution of ammonium chloride ( $5 \mathrm{~mL})$. The solution was allowed to warm to room temperature and then the layers were separated. The aqueous layer was extracted with dichloromethane $(3 \times 5 \mathrm{~mL})$ and the combined organic layers were dried over $\mathrm{MgSO}_{4}$ and concentrated in vacuo. The residue was purified by flash column chromatography with cyclohexane/ethyl acetate (19:1-9:1, $\mathrm{v} / \mathrm{v}$ ) as eluent and give 25 as an oil $(1.21 \mathrm{~g}, 83 \%) . R_{f} .0 .47(\mathrm{cy}-$ clohexane/AcOEt $=4: 1) ;[\alpha]_{\mathrm{D}}^{25}-22.8\left(c 1.8, \mathrm{CHCl}_{3}\right)$; IR (film): $\nu$ 3518, 1747, 1245-1025; ${ }^{1} \mathrm{H}$ NMR $(400 \mathrm{MHz}$, $\left.\mathrm{CDCl}_{3}\right): \delta 7.68-7.63(\mathrm{~m}, 4 \mathrm{H}, \mathrm{H}-\mathrm{Ar}), 7.47-7.38(\mathrm{M}, 6 \mathrm{H}, \mathrm{H}-$ Ar), $4.26\left(\mathrm{dt},{ }^{3} J=8.0\right.$ and $3.0 \mathrm{~Hz}, 1 \mathrm{H}, \mathrm{CHO}$ ), 3.99 (dd, $\left.{ }^{2} J=10.5 \mathrm{~Hz},{ }^{3} J=3.0 \mathrm{~Hz}, 1 \mathrm{H}, \mathrm{CH}_{2} \mathrm{O}\right), 3.94\left(\mathrm{dd},{ }^{2} J=10.5 \mathrm{~Hz}\right.$, $\left.{ }^{3} J=3.0 \mathrm{~Hz}, 1 \mathrm{H}, \mathrm{CH}_{2} \mathrm{O}\right), 3.80(\mathrm{~s}, 3 \mathrm{H}, \mathrm{Me}), 3.18\left(\mathrm{~d},{ }^{3} J=\right.$ $8.0 \mathrm{~Hz}, 1 \mathrm{H}, \mathrm{OH}), 1.05(\mathrm{~s}, 9 \mathrm{H}, \mathrm{Me}) ;{ }^{13} \mathrm{C}$ NMR $(100 \mathrm{MHz}$, $\left.\mathrm{CDCl}_{3}\right): \delta 173.2(\mathrm{CO}), 135.5(\mathrm{C}-\mathrm{Ar}), 132.9$ and $132.8(\mathrm{C}-$ $\mathrm{Ar}), 129.8$ (C-Ar), 127.7 (C-Ar), 71.9 (CHO), 65.8 $\left(\mathrm{CH}_{2} \mathrm{O}\right), 52.4(\mathrm{Me}), 26.6(\mathrm{Me}), 19.2\left(\mathrm{C}-\left(\mathrm{CH}_{3}\right)_{3}\right)$.

4.2.19. (+)-Methyl-(2R)-3-(tert-butyldiphenylsilyloxy)2-(methoxymethoxy)propanoate (26). To a solution of $\mathbf{2 5}$ $(8.50 \mathrm{~g}, 23.7 \mathrm{~mol})$ in $\mathrm{CH}_{2} \mathrm{Cl}_{2}(55 \mathrm{~mL})$ were added, at $20^{\circ} \mathrm{C}$ and under argon, diisopropylethylamine $(12.4 \mathrm{~mL}$, $71.1 \mathrm{mmol})$ and chloromethylmethylether $(5.4 \mathrm{~mL}$, $71.1 \mathrm{mmol}$ ). The resulting mixture was stirred overnight at $20^{\circ} \mathrm{C}$. Then were added more diisopropylethylamine $(4.1 \mathrm{~mL}, 23.7 \mathrm{mmol})$ and chloromethylmethylether $(1.8 \mathrm{~mL}$, $23.7 \mathrm{mmol}$ ). After $8 \mathrm{~h}$ at $20^{\circ} \mathrm{C}$, the reaction was finally completed and quenched by water $(16 \mathrm{~mL})$. The layers were separated and the organic one was washed with water $(16 \mathrm{~mL})$, dried $\left(\mathrm{MgSO}_{4}\right)$ and concentrated. Purification by column chromatography using cyclohexane/ethyl acetate $(39: 1, \mathrm{v} / \mathrm{v})$ as eluent gave $26(8.04 \mathrm{~g}, 84 \%) . R_{f} 0.51$ (cyclohexane/ethyl acetate $=4: 1) ;[\alpha]_{\mathrm{D}}^{25}+7.0\left(c 1.3, \mathrm{CHCl}_{3}\right) ; \mathrm{IR}($ film $): \nu 1752$, $1260-1045 \mathrm{~cm}^{-1}$; ${ }^{1} \mathrm{H}$ NMR $\left(400 \mathrm{MHz}, \mathrm{CDCl}_{3}\right): \delta 7.71-$ 7.67 (m, 4H, H-Ar), 7.46-7.36 (M, 6H, H-Ar), 4.73 (s, 
$2 \mathrm{H}, \mathrm{O}-\mathrm{CH}_{2}-\mathrm{O}$ ), $4.31\left(\mathrm{dd},{ }^{3} \mathrm{~J}=5.5 \mathrm{~Hz},{ }^{3} \mathrm{~J}=4.5 \mathrm{~Hz}, 1 \mathrm{H}\right.$, CHO), 3.97 (dd, ${ }^{2} J=10.5 \mathrm{~Hz},{ }^{3} J=5.5 \mathrm{~Hz}, 1 \mathrm{H}, \mathrm{CH}_{2} \mathrm{O}$ ), 3.94 (dd, ${ }^{2} J=10.5 \mathrm{~Hz},{ }^{3} J=4.5 \mathrm{~Hz}, 1 \mathrm{H}, \mathrm{CH}_{2} \mathrm{O}$ ), 3.75 (s, $3 \mathrm{H}, \mathrm{Me}$ ), 3.37 (s, 3H, Me), 1.05 (s, 9H, Me); ${ }^{13} \mathrm{C}$ NMR (100 MHz, $\left.\mathrm{CDCl}_{3}\right): \delta 171.1(\mathrm{CO}), 135.6$ and $135.5(\mathrm{C}-\mathrm{Ar}), 133.1$ and 133.0 (C-Ar), 129.7 (C-Ar), $127.7(\mathrm{C}-\mathrm{Ar}), 96.2\left(\mathrm{O}-\mathrm{CH}_{2}-\right.$ O), $76.5(\mathrm{CHO}), 64.7\left(\mathrm{CH}_{2} \mathrm{O}\right), 55.8\left(\mathrm{CH}_{3}\right), 51.9\left(\mathrm{CH}_{3}\right)$, $26.6\left(\mathrm{CH}_{3}\right), 19.2\left(\mathrm{C}-\left(\mathrm{CH}_{3}\right)_{3}\right)$; HRMS (ESI) calcd for $\mathrm{C}_{22} \mathrm{H}_{30} \mathrm{O}_{5} \mathrm{SiNa}[\mathrm{M}+\mathrm{Na}]^{+}: 425.1760$, found: 425.1757 .

4.2.20. (2R)-3-(tert-Butyldiphenylsilyloxy)-2-(methoxymethoxy)propanoyl chloride (27). To a solution of ester $26(6.04 \mathrm{~g}, 15.0 \mathrm{mmol})$ in tetrahydrofuran $/$ methanol (420 mL, 4:1, v/v) was added, under argon, a $1 \mathrm{M}$ solution of lithium hydroxide $(60 \mathrm{~mL})$ in $\mathrm{THF} / \mathrm{MeOH}(4: 1, \mathrm{v} / \mathrm{v})$. The reaction mixture was stirred $4 \mathrm{~h}$ at $20^{\circ} \mathrm{C}$ and then the solvents were evaporated in vacuo. Traces of water were eliminated by washing the residue twice with anhydrous toluene. The lithium salt of 26 was characterized by its ${ }^{1} \mathrm{H}$ NMR spectrum. ${ }^{1} \mathrm{H}$ NMR $\left(400 \mathrm{MHz}, \mathrm{CD}_{3} \mathrm{OD}\right): \delta 7.76-$ $7.71(\mathrm{~m}, 4 \mathrm{H}, \mathrm{H}-\mathrm{Ar}), 7.43-7.35(\mathrm{M}, 6 \mathrm{H}, \mathrm{H}-\mathrm{Ar}), 4.76$ (d, $\left.{ }^{2} J=7.0 \mathrm{~Hz}, 1 \mathrm{H}, \mathrm{O}-\mathrm{CH}_{2}-\mathrm{O}\right), 4.74\left(\mathrm{~d},{ }^{2} J=7.0 \mathrm{~Hz}, 1 \mathrm{H}, \mathrm{O}-\right.$ $\left.\mathrm{CH}_{2}-\mathrm{O}\right), 4.26\left(\mathrm{dd},{ }^{3} \mathrm{~J}=7.5\right.$ and $\left.3.0 \mathrm{~Hz}, 1 \mathrm{H}, \mathrm{CHO}\right), 3.98$ (dd, ${ }^{2} J=10.5 \mathrm{~Hz},{ }^{3} J=3.0 \mathrm{~Hz}, \quad 1 \mathrm{H}, \mathrm{CH}_{2} \mathrm{O}$ ), 3.88 (dd, ${ }^{2} J=10.5 \mathrm{~Hz},{ }^{3} J=7.5 \mathrm{~Hz}, 1 \mathrm{H}, \mathrm{CH}_{2} \mathrm{O}$ ), 3.40 (s, $3 \mathrm{H}, \mathrm{Me}$ ), $1.04(\mathrm{~s}, 9 \mathrm{H}, \mathrm{Me})$. The lithium salt of $26(2.5 \mathrm{mmol})$ was dissolved, under argon at $0{ }^{\circ} \mathrm{C}$, in anhydrous ether $(10 \mathrm{~mL})$ before adding pyridine $(55 \mu \mathrm{L}, 0.5 \mathrm{mmol})$ followed by freshly distilled oxalyl chloride $(430 \mu \mathrm{L}, 5.0 \mathrm{mmol})$. The reaction mixture was stirred overnight. Elimination of the solvent furnished quantitatively acyl chloride 27 .

4.2.21. (+)- $N, N^{\prime}-\{2-[($ Benzyloxy $)$ imino]propane-1,3diyl\}bis[(2R)-3-(tert-butyldiphenylsilyloxy)-2-(methoxymethoxy)propanamide] (28). Amine (12) (1.13 mmol) was dissolved, under argon and at $0{ }^{\circ} \mathrm{C}$, into $\mathrm{CH}_{2} \mathrm{Cl}_{2}(7 \mathrm{~mL})$. Triethylamine $(1.0 \mathrm{~mL}, 7.47 \mathrm{mmol})$ and 4-dimethylaminopyridine $(0.05 \mathrm{~g}, 0.45 \mathrm{mmol})$ were then added followed, after $15 \mathrm{~min}$, by 27 ( $1.02 \mathrm{~g}, 2.49 \mathrm{mmol})$. The stirring was continued for $18 \mathrm{~h}$. The reaction mixture was diluted with $\mathrm{CH}_{2} \mathrm{Cl}_{2}(55 \mathrm{~mL})$. The solution was washed twice with water $(16 \mathrm{~mL})$ followed by a saturated solution of $\mathrm{Na}_{2} \mathrm{CO}_{3}(8 \mathrm{~mL})$ and dried $\left(\mathrm{MgSO}_{4}\right)$. Purification of the crude residue by flash column chromatography with cyclohexane/ethyl acetate $(7: 3, \mathrm{v} / \mathrm{v})$ as eluent gave 28 as a gum $(0.39 \mathrm{~g}, 37 \%) . R_{f}$ : 0.30 (cyclohexane/ethyl acetate $=7: 3$ ); $[\alpha]_{\mathrm{D}}^{25}+19.7$ ( c 1.2 , $\mathrm{CHCl}_{3}$ ); IR (film) $\nu 3425,3322,1680,1110-1028 \mathrm{~cm}^{-1}$; ${ }^{1} \mathrm{H}$ NMR (400 MHz, $\left.\mathrm{CDCl}_{3}\right): \delta 7.69-7.63(\mathrm{M}, 8 \mathrm{H}, \mathrm{H}-\mathrm{Ar})$, 7.42-7.30 (M, 19H, H-Ar and $\mathrm{NH}), 5.06\left(\mathrm{~s}, 2 \mathrm{H}, \mathrm{CH}_{2} \mathrm{Ph}\right)$, $4.74\left(\mathrm{~d},{ }^{2} J=6.5 \mathrm{~Hz}, 1 \mathrm{H}, \mathrm{O}-\mathrm{CH}_{2}-\mathrm{O}\right), 4.69\left(\mathrm{~d},{ }^{2} J=6.5 \mathrm{~Hz}, 1 \mathrm{H}\right.$, $\left.\mathrm{O}-\mathrm{CH}_{2}-\mathrm{O}\right), 4.67\left(\mathrm{~d},{ }^{2} J=6.5 \mathrm{~Hz}, 1 \mathrm{H}, \mathrm{O}-\mathrm{CH}_{2}-\mathrm{O}\right), 4.63$ (d, $\left.{ }^{2} J=6.5 \mathrm{~Hz}, 1 \mathrm{H}, \mathrm{O}-\mathrm{CH}_{2}-\mathrm{O}\right), 4.26-4.18(\mathrm{M}, 3 \mathrm{H}, \mathrm{CHO}$ and $\mathrm{H}-\mathrm{c}), 4.14$ (dd, $\left.{ }^{2} J=12.0 \mathrm{~Hz},{ }^{3} J=6.0 \mathrm{~Hz}, 1 \mathrm{H}, \mathrm{H}-\mathrm{a}\right), 4.10$ (dd, $\left.{ }^{2} J=11.0 \mathrm{~Hz},{ }^{3} J=6.0 \mathrm{~Hz}, 1 \mathrm{H}, \mathrm{H}-\mathrm{c}\right), 4.01-3.93$ (M, $5 \mathrm{H}$, $\mathrm{CH}_{2} \mathrm{OSi}$ and $\mathrm{H}-\mathrm{a}$ ), 3.32, (s, 3H, H-Me), 3.29 (s, 3H, Me), $1.02(\mathrm{~s}, 18 \mathrm{H}, \mathrm{Me}) ;{ }^{13} \mathrm{C}$ NMR $\left(100 \mathrm{MHz}, \mathrm{CDCl}_{3}\right): \delta 170.4$ (CO), $170.1(\mathrm{CO}), 153.1$ (C-b), $137.2(\mathrm{C}-\mathrm{Ar}), 135.6(\mathrm{C}-\mathrm{Ar})$, 133.1 (C-Ar), 133.0 (C-Ar), 129.7 (C-Ar), 128.4 (CAr), 128.2 (C-Ar), 128.0 (C-Ar), 127.7 (C-Ar), 96.2 (C$\mathrm{Ar}), 78.4(\mathrm{CHO}), 78.3(\mathrm{CHO}), 76.5\left(\mathrm{CH}_{2} \mathrm{Ph}\right), 65.0\left(\mathrm{CH}_{2} \mathrm{O}\right)$, $64.7\left(\mathrm{CH}_{2} \mathrm{O}\right), 56.0\left(\mathrm{CH}_{3}\right), 55.9\left(\mathrm{CH}_{3}\right), 40.3(\mathrm{C}-\mathrm{a}), 35.3$ (C-c), $26.7\left(\mathrm{CH}_{3}\right), 19.2\left(\mathrm{CH}_{3}\right)$; HRMS (ESI): calcd for $\mathrm{C}_{52} \mathrm{H}_{67} \mathrm{~N}_{3} \mathrm{O}_{9} \mathrm{Si}_{2} \mathrm{Na}[\mathrm{M}+\mathrm{Na}]^{+}:$956.4314, found: 956.4340 .
4.2.22. (+)- $N, N^{\prime}-\{2-[($ Benzyloxy $)$ imino $]$ propane-1,3diyl\}-bis [(2R)-3-(tert-butyldiphenylsilyloxy)-2-hydroxypropanamide $](29)$ and $(+)-N, N^{\prime}-(2-0 x o p r o p a n e-1,3-$ diyl)bis[(2R)-3-(tert-butyldiphenylsilyloxy)-2-hydroxypropanamide] (30b). Method A: to a solution of oxime 28 (950 mg, $1.01 \mathrm{mmol})$ in anhydrous $\mathrm{CH}_{2} \mathrm{Cl}_{2}(35 \mathrm{~mL})$ was added, at $0{ }^{\circ} \mathrm{C}$ and under argon, trimethylsilylbromide $(1.07 \mathrm{~mL}, 8.11 \mathrm{mmol})$. The reaction mixture was stirred for $4 \mathrm{~h}$. After quenching by adding a saturated solution of $\mathrm{NaHCO}_{3}(30 \mathrm{~mL})$, the layers were separated. The aqueous layer was extracted with $\mathrm{CH}_{2} \mathrm{Cl}_{2}$. The combined organic layers were dried $\left(\mathrm{MgSO}_{4}\right)$ and concentrated under vacuo. The residue was purified by flash column chromatography using cyclohexane/ethyl acetate $(3: 2-1: 4)$ as eluent and gave $(R, R)-29(0.460 \mathrm{~g}, 54 \%)$ and $(R, R)-30(0.150 \mathrm{~g}, 20 \%)$.

Method B: to a solution of $29(0.344 \mathrm{~g}, 0.41 \mathrm{mmol})$ in acetone/water $(8 \mathrm{~mL}, 10 / 1, \mathrm{v} / \mathrm{v})$, were added Amberlyst ${ }^{\circledR} 15$ $(0.100 \mathrm{~g})$ and paraformaldehyde $(0.120 \mathrm{~g}, 4.07 \mathrm{mmol})$. The resulting mixture was heated under reflux for $24 \mathrm{~h}$. After filtration on a Celite ${ }^{\circledR}$ pad and concentration, the residue was purified by flash column chromatography with cyclohexane/ethyl acetate $(3: 2-1: 4)$ as eluent and gave $29(0.130 \mathrm{~g}$, $38 \%)$ and $30(0.078 \mathrm{~g}, 26 \%)$.

Compound (+)-(R,R)-(29). $\quad R_{f}: 0.45 \quad$ (cyclohexane/ethyl acetate $=3: 2) ;[\alpha]_{\mathrm{D}}^{25}+12.1\left(\right.$ c $\left.5.6, \mathrm{CHCl}_{3}\right) ;$ IR (film): $\nu$ $3396,1666 \mathrm{~cm}^{-1} ;{ }^{1} \mathrm{H}$ NMR $\left(400 \mathrm{MHz}, \mathrm{CDCl}_{3}\right): \delta 7.69-$ $7.64(\mathrm{M}, 8 \mathrm{H}, \mathrm{H}-\mathrm{Ar}), 7.49-7.30(\mathrm{M}, 19 \mathrm{H}, \mathrm{H}-\mathrm{Ar}$ and $\mathrm{NH})$, $5.10\left(\mathrm{~s}, 2 \mathrm{H}, \mathrm{CH}_{2} \mathrm{Ph}\right), 4.27-4.13(\mathrm{M}, 5 \mathrm{H}, \mathrm{CHO}, \mathrm{H}-\mathrm{a}$ and $\mathrm{H}-$ c), 3.99-3.90 (M, $5 \mathrm{H}, \mathrm{CH}_{2} \mathrm{O}$ and $\left.\mathrm{H}-\mathrm{a}\right), 3.51\left(\mathrm{~d},{ }^{3} \mathrm{~J}=5.0 \mathrm{~Hz}\right.$, $1 \mathrm{H}, \mathrm{OH}), 3.50\left(\mathrm{~d},{ }^{3} J=5.0 \mathrm{~Hz}, 1 \mathrm{H}, \mathrm{OH}\right), 1.07(\mathrm{~s}, 18 \mathrm{H}, \mathrm{Me})$; ${ }^{13} \mathrm{C}$ NMR (100 MHz, $\left.\mathrm{CDCl}_{3}\right): \delta 172.2(\mathrm{CO}), 172.0(\mathrm{CO})$, 153.7 (C-b), 137.1 (C-Ar), 135.4 (C-Ar), 132.7 (C-Ar), 132.6 (C-Ar), 129.9 (C-Ar), 128.4 (C-Ar), 128.2 (C-Ar), 128.0 (C-Ar), 127.8 (C-Ar), $76.5\left(\mathrm{CH}_{2} \mathrm{Ph}\right), 72.4(\mathrm{CHO})$, 72.3 (CHO), $65.15\left(\mathrm{CH}_{2} \mathrm{O}\right), 65.1\left(\mathrm{CH}_{2} \mathrm{O}\right), 39.9(\mathrm{C}-\mathrm{a}), 35.2$ (C-c), $26.7\left(\mathrm{CH}_{3}\right), 19.2\left(\mathrm{CH}_{3}\right)$; HRMS (ESI): calcd for $\mathrm{C}_{48} \mathrm{H}_{59} \mathrm{~N}_{3} \mathrm{O}_{7} \mathrm{Si}_{2} \mathrm{Na}[\mathrm{M}+\mathrm{Na}]^{+}:$868.3789, found: 868.3792.

Compound (+)-(R,R)-(30b). $R_{f}: 0.12$ (cyclohexane/ethyl acetate $=3: 2) ;[\alpha]_{\mathrm{D}}^{25}+10.5\left(c 1.8, \mathrm{CHCl}_{3}\right)$; IR (film): $\nu$ $3397,1665,1111 \mathrm{~cm}^{-1}$; ${ }^{1} \mathrm{H}$ NMR $\left(400 \mathrm{MHz}, \mathrm{CDCl}_{3}\right)$ : $\delta$ 7.66-7.62 (M, 8H, H-Ar), 7.46-7.36 (M, 14H, H-Ar and $\mathrm{NH}), 4.23$ (dd, ${ }^{2} J=19.0 \mathrm{~Hz},{ }^{3} J=5.5 \mathrm{~Hz}, 2 \mathrm{H}, \mathrm{H}-\mathrm{a}$ and $\mathrm{H}-\mathrm{c}$ ), $4.23(\mathrm{~m}, 2 \mathrm{H}, \mathrm{CHO}), 4.16\left(\mathrm{dd},{ }^{2} J=19.0 \mathrm{~Hz},{ }^{3} J=5.0 \mathrm{~Hz}, 2 \mathrm{H}\right.$, $\mathrm{H}-\mathrm{a}$ and $\mathrm{H}-\mathrm{c}$ ), 3.93 (dd, ${ }^{2} J=10.5 \mathrm{~Hz},{ }^{3} J=5.0 \mathrm{~Hz}, 2 \mathrm{H}, \mathrm{CH}_{2} \mathrm{O}$ ), $3.91\left(\mathrm{dd},{ }^{2} J=11.0 \mathrm{~Hz},{ }^{3} J=5.0 \mathrm{~Hz}, 2 \mathrm{H}, \mathrm{CH}_{2} \mathrm{O}\right), 3.38(\mathrm{se}, 2 \mathrm{H}$, $\mathrm{OH}), 1.06(\mathrm{~s}, 18 \mathrm{H}, \mathrm{Me}) ;{ }^{13} \mathrm{C}$ NMR $\left(100 \mathrm{MHz}, \mathrm{CDCl}_{3}\right)$ $\delta 199.9$ (C-b), 172.0 (CO), 135.5 and 135.4 (C-Ar), 132.6 and $132.4(\mathrm{C}-\mathrm{Ar}), 130.0(\mathrm{C}-\mathrm{Ar}), 127.9(\mathrm{C}-\mathrm{Ar}), 71.9$ (CHO), $65.1\left(\mathrm{CH}_{2} \mathrm{O}\right), 46.8(\mathrm{C}-\mathrm{a}$ and $\mathrm{C}-\mathrm{c}), 26.8\left(\mathrm{CH}_{3}\right), 19.2$ $\left(\mathrm{CH}_{3}\right)$. HRMS (ESI): calcd for $\mathrm{C}_{41} \mathrm{H}_{52} \mathrm{~N}_{2} \mathrm{O}_{7} \mathrm{Si}_{2} \mathrm{Na}$ $[\mathrm{M}+\mathrm{Na}]^{+}:$763.3211, found: 763.3181 .

4.2.23. (+)-(2R)-3-(tert-Butyldiphenylsilyloxy)-2-hydroxy- $N-(\{(6 R)-2$-butoxy-6-[(tert-butyldiphenylsilyloxy)methyl]-5-oxomorpholin-2-yl\}methyl)propanamide (31) and (-)-(2R)-3-(tert-Butyldiphenylsilyloxy)-2-hydroxy$N$-(\{(2R)-2-[(tert-butyldiphenylsilyloxy)methyl]-3-oxo2,3-dihydro-2H-1,4-oxazin-6-yl \}methyl)propanamide (32). A solution of $30 \mathrm{~b}(0.078 \mathrm{~g}, 0.105 \mathrm{mmol})$ and $p \mathrm{TsOH}$ $(0.001 \mathrm{~g})$ in 1-butanol $(2.5 \mathrm{~mL})$ were heated under reflux 
for $6 \mathrm{~h}$. After concentration a mixture of starting material $\mathbf{3 0 b}$, compound $\mathbf{3 1}$ and compound $\mathbf{3 2}$, which could be easily separated by flash column chromatography was obtained. These three new compounds could be fully characterized at this stage. A mixture of $\mathbf{3 0 b}, \mathbf{3 1}$ and $\mathbf{3 2}$ was then dissolved in toluene $(2.4 \mathrm{~mL})$ and heated under reflux for $5 \mathrm{~h}$. After evaporation of the solvent the crude residue was flash chromatographed with cyclohexane/ethyl acetate $(1: 1, \mathrm{v} / \mathrm{v})$ as eluent and gave $(R, R)-31(0.044 \mathrm{~g}, 57 \%)$.

Compound (+)-(R,R)-(31). Gum; $R_{f}: 0.50$ (cyclohexane/ ethyl acetate $=1: 1) ;[\alpha]_{\mathrm{D}}^{25}+25.9\left(c 0.2, \mathrm{CHCl}_{3}\right)$; IR (film): $\nu$ $3412,1682,1113 \mathrm{~cm}^{-1}$; ${ }^{1} \mathrm{H}$ NMR $\left(400 \mathrm{MHz}, \mathrm{CDCl}_{3}\right)$ : $\delta 7.69(\mathrm{~m}, 4 \mathrm{H}, \mathrm{H}-\mathrm{Ar}), 7.62(\mathrm{~m}, 4 \mathrm{H}, \mathrm{H}-\mathrm{Ar}), 7.48-7.35$ (M, $12 \mathrm{H}, \mathrm{H}-\mathrm{Ar}), 6.94\left(\mathrm{t},{ }^{3} \mathrm{~J}=6.5 \mathrm{~Hz}, 1 \mathrm{H}, \mathrm{NH}\right), 5.60\left(\mathrm{~d},{ }^{3} \mathrm{~J}=\right.$ $4.5 \mathrm{~Hz}, 1 \mathrm{H}, \mathrm{NH}$-cycle), 4.19 (dd, ${ }^{3} J=4.0 \mathrm{~Hz},{ }^{3} J=2.0 \mathrm{~Hz}$, $1 \mathrm{H}, \mathrm{CHO}), 4.17-4.11(\mathrm{M}, 2 \mathrm{H}, \mathrm{CHOH}$ and $\mathrm{CH}$-cycle$\mathrm{CH}_{2} \mathrm{O}$ ), 3.99 (dd, ${ }^{2} J=10.5 \mathrm{~Hz},{ }^{3} J=2.0 \mathrm{~Hz}, 1 \mathrm{H}, \mathrm{CH}$-cycle$\mathrm{CH}_{2} \mathrm{O}$ ), 3.98 (dd, ${ }^{2} \mathrm{~J}=10.5 \mathrm{~Hz},{ }^{3} \mathrm{~J}=5.0 \mathrm{~Hz}, 1 \mathrm{H}, \mathrm{CH}-\mathrm{CH}_{2} \mathrm{O}$ ), 3.93 (dd, ${ }^{2} J=10.5 \mathrm{~Hz},{ }^{3} \mathrm{~J}=4.5 \mathrm{~Hz}, 1 \mathrm{H}, \mathrm{CH}-\mathrm{CH}_{2} \mathrm{O}$ ), 3.66 (dd, $\left.{ }^{2} J=14.0 \mathrm{~Hz},{ }^{3} J=7.0 \mathrm{~Hz}, 1 \mathrm{H},-\mathrm{CH}_{2} \mathrm{NH}\right), 3.58-3.46(\mathrm{M}, 4 \mathrm{H}$, $-\mathrm{CH}_{2} \mathrm{NH},-\mathrm{CH}_{2}$-cycle-NH and $\mathrm{OCH}_{2} \mathrm{CH}_{2}-$ ), 3.23 (dd, ${ }^{2} J=12.5 \mathrm{~Hz},{ }^{3} J=5.0 \mathrm{~Hz}, 1 \mathrm{H},-\mathrm{CH}_{2}$-cycle-NH), 3.02 (de, $\left.{ }^{3} J=4.5 \mathrm{~Hz}, 1 \mathrm{H}, \mathrm{OH}\right), 1.55\left(\mathrm{Q},{ }^{2} J=7.0 \mathrm{~Hz}, 2 \mathrm{H},-\mathrm{OCH}_{2} \mathrm{CH}_{2}\right)$, $1.36\left(\mathrm{~h},{ }^{3} \mathrm{~J}=7.5 \mathrm{~Hz}, 2 \mathrm{H}, \mathrm{CH}_{3} \mathrm{CH}_{2}\right), 1.07$ (s, 9H, Me), $1.03(\mathrm{~s}$, $9 \mathrm{H}, \mathrm{Me}), 0.91\left(\mathrm{t},{ }^{3} \mathrm{~J}=7.5 \mathrm{~Hz}, 3 \mathrm{H}, \mathrm{Me}\right) ;{ }^{13} \mathrm{C}$ NMR $(100 \mathrm{MHz}$, $\mathrm{CDCl}_{3}$ ): $\delta 171.7$ (NHCO), 168.2 (NHCO-cycle), $135.7-$ 135.6-135.4 (C-Ar), 133.4-133.1-132.5 (C-Ar), 130.1129.7 (C-Ar), 127.9-127.7 (C-Ar), 96.1 (O-C-O), 74.1 $\left(\mathrm{CH}\right.$-cycle-O), $72.0(\mathrm{CH}-\mathrm{O}), 65.1\left(\mathrm{CH}_{2}-\mathrm{O}\right), 64.2(\mathrm{CH}-$ cycle- $\left.\mathrm{CH}_{2}-\mathrm{O}\right), 61.4\left(-\mathrm{O}-\mathrm{CH}_{2} \mathrm{CH}_{2}-\right), 47.4\left(\mathrm{~N}-\mathrm{CH}_{2}-\right.$ cycle- $)$, $41.3\left(\mathrm{NH}-\mathrm{CH}_{2}-\right), 31.8\left(\mathrm{O}-\mathrm{CH}_{2} \mathrm{CH}_{2}-\right), 26.9-26.7\left(\mathrm{CH}_{3}\right)$, $19.4\left(\mathrm{CH}_{3} \mathrm{CH}_{2}-\right), 19.3\left(\mathrm{C}-\left(\mathrm{CH}_{3}\right)_{3}\right), 13.9\left(\mathrm{CH}_{3}\right)$; HRMS (ESI): calcd for $\mathrm{C}_{45} \mathrm{H}_{60} \mathrm{~N}_{2} \mathrm{O}_{7} \mathrm{Si}_{2} \mathrm{Na}[\mathrm{M}+\mathrm{Na}]^{+}$: 819.3837 , found: 819.3867

Compound (-)- $(R, R)-(32) . R_{f}=0.45$ (ethyl acetate/cyclohexane $=3: 2) ;[\alpha]_{\mathrm{D}}^{25}-10.3\left(c 0.2, \mathrm{CHCl}_{3}\right) ; \mathrm{IR}$ (film): $\nu$ $3392,1682,1113 \mathrm{~cm}^{-1} ;{ }^{1} \mathrm{H}$ NMR $\left(400 \mathrm{MHz}, \mathrm{CDCl}_{3}\right)$ : $\delta$ 7.70-7.61 (M, 8H, H-Ar), 7.46-7.35 (M, 13H, H-Ar and NH-cycle-), $7.06\left(\mathrm{t},{ }^{3} J=5.5 \mathrm{~Hz}, 1 \mathrm{H}, \mathrm{NH}\right), 5.59\left(\mathrm{~d},{ }^{3} J=\right.$ $5.0 \mathrm{~Hz}, 1 \mathrm{H}, \mathrm{CH}-\mathrm{NH}$-cycle-), 4.54 (dd, ${ }^{3} \mathrm{~J}=4.5$ and $2.5 \mathrm{~Hz}$, $1 \mathrm{H}, \mathrm{O}-\mathrm{CH}$-cycle-), 4.14 (dd, ${ }^{2} J=11.0 \mathrm{~Hz},{ }^{3} J=4.5 \mathrm{~Hz}, 1 \mathrm{H}$, $\mathrm{CH}$-cycle- $\mathrm{CH}_{2}$ ), 4.13 (t, ${ }^{3} \mathrm{~J}=5.5 \mathrm{~Hz}, 1 \mathrm{H}, \mathrm{CH}-\mathrm{O}$ ), 3.97 (dd, ${ }^{2} \mathrm{~J}=11.5 \mathrm{~Hz},{ }^{3} \mathrm{~J}=2.5 \mathrm{~Hz}, 1 \mathrm{H}$, CH-cycle- $\left.\mathrm{CH}_{2}\right), 3.94-3.85$ $\left(\mathrm{M}, 4 \mathrm{H}, \mathrm{CH} 2 \mathrm{NH}\right.$ and $\left.\mathrm{CH}-\mathrm{CH}_{2} \mathrm{O}\right), 3.15\left(\mathrm{~d},{ }^{3} \mathrm{~J}=4.5 \mathrm{~Hz}, 1 \mathrm{H}\right.$, $\mathrm{OH}), 1.06(\mathrm{~s}, 9 \mathrm{H}, \mathrm{Me}), 1.04(\mathrm{~s}, 9 \mathrm{H}, \mathrm{Me}) ;{ }^{13} \mathrm{C} \mathrm{NMR}$ $\left(100 \mathrm{MHz}, \mathrm{CDCl}_{3}\right): \delta 171.4(-\mathrm{NH}-\mathrm{CO}-), 163.8(-\mathrm{NH}-\mathrm{CO}-$ cycle), $136.0(\mathrm{O}-\mathrm{C}=\mathrm{CH}), 135.6$ and $135.5(\mathrm{C}-\mathrm{Ar}), 135.5$ and $135.4(\mathrm{C}-\mathrm{Ar}), 133.1$ and 132.9 (C-Ar), 132.6 and 132.4 (C-Ar), 130.0 (C-Ar), 129.8 (C-Ar), 127.9 (C-Ar), $127.7(\mathrm{C}-\mathrm{Ar}), 102.1(\mathrm{C}=\mathrm{CH}), 78.0\left(\mathrm{CH}-\mathrm{cycle}-\mathrm{CH}_{2}\right), 71.8$ $(\mathrm{CH}-\mathrm{O}), 65.1$ (-CH-CH$-\mathrm{C}), 64.1$ ( $\mathrm{CH}-$ cycle- $\left.\mathrm{CH}_{2}-\right), 38.8$ $\left(\mathrm{CH}_{2} \mathrm{NH}\right), 26.8\left(\mathrm{CH}_{3}\right), 26.6\left(\mathrm{CH}_{3}\right), 19.2\left(\mathrm{C}-\left(\mathrm{CH}_{3}\right)_{3}\right)$; HRMS (ESI): calcd for $\mathrm{C}_{41} \mathrm{H}_{50} \mathrm{~N}_{2} \mathrm{O}_{6} \mathrm{Si}_{2} \mathrm{Na}[\mathrm{M}+\mathrm{Na}]^{+}$: 745.3105 , found: 745.3133 .

\section{References and notes}

1. For reviews, see: (a) Mead, K. T.; Brewer, B. N. Curr. Org. Chem. 2003, 7, 227-256; (b) Francke, W.; Kitching, W. Curr. Org. Chem. 2001, 5, 233-251.
2. (a) Deslongchamps, P.; Rowan, D. D.; Pothier, N.; Sauvé, G.; Saunders, J. K. Can. J. Chem. 1981, 59, 1105-1121; (b) Pothier, N.; Goldstein, S.; Deslongchamps, P. Helv. Chim. Acta 1992, 75, 604-620.

3. Yin, B.-L.; Yang, Z.-M.; Hu, T.-S.; Wu, Y.-L. Synthesis 2003, 13, 1995-2000.

4. Hossain, N.; Zapata, A.; Wilstermann, M.; Nilsson, U. J.; Magnusson, G. Carbohydr. Res. 2002, 337, 569-580.

5. (a) Seward, E. M.; Carlson, E.; Harrison, T.; Haworth, K. E.; Herbert, R.; Kelleher, F. J.; Kurtz, M. M.; Moseley, J.; Owen, S. N.; Owens, A. P.; Sadowski, S. J.; Swain, C. J.; Williams, B. J. Bioorg. Med. Chem. Lett. 2002, 12, 2515-2518; (b) Williams, B. J.; Cascieri, M. A.; Chicchi, G. G.; Harrison, T.; Owens, A. P.; Owen, S. N.; Rupniak, N. M. J.; Tattersall, D. F.; Williams, A.; Swain, C. J. Bioorg. Med. Chem. Lett. 2002, 12, 2719-2722; (c) Raubo, P.; Kulagowski, J. J.; Swain, C. J. Synlett 2003, 2021-2024.

6. Trump, R. P.; Bartlett, P. A. J. Comb. Chem. 2003, 5, 285-291.

7. Goubert, M.; Canet, I.; Sinibaldi, M.-E. Eur. J. Org. Chem. 2006, 23, 4805-4812.

8. Amine 6 was classically prepared from commercially available solketal in two steps and 79\% overall yield via its phthalimide derivative (Lohray, B. B.; Sekar Reddy, A.; Bhushan, V. Tetrahedron: Asymmetry 1996, 7, 2411-2416).

9. Amine 7 was obtained from the mesylate of solketal (Kim, H. S.; Barak, D.; Harden, T. K.; Boyer, J. L.; Jacobson, K. A. J. Med. Chem. 2001, 44, 3092-3108) by treatment with benzylamine in $\mathrm{CH}_{3} \mathrm{CN}$ as previously mentionned by; Lemaire, $\mathrm{M}$.; Posada, F.; Gourcy, J.-G.; Jeminet, G. Synthesis 1995, 6, 627-629.

10. For facilities, all new oximes prepared and described in the experimental part were depicted using the same numeroation we indicated in Scheme 1.

11. Ryu, I.; Kuriyama, H.; Minakata, S.; Komatsu, M.; Yoon, J. Y.; Kim, S. J. Am. Chem. Soc. 1999, 121, 12190-12191.

12. Das, N. B.; Nanda, B.; Nayak, A. Synth. Commun. 2002, 32, $3647-3651$

13. Xiao, X.; Bai, D. Synlett 2001, 535-537.

14. (a) Tsuritani, T.; Yagi, K.; Shinokubo, H.; Oshima, K. Angew. Chem., Int. Ed. 2003, 42, 5613-5615; (b) Kaiser, A.; Wiegrebe, W. Monatsh. Chem. 1996, 127, 763-774; (c) Blake, J. A.; Ingold, K. U.; Lin, S.; Mulder, P.; Pratt, D. A.; Sheeller, B.; Walton, J. C. Org. Biomol. Chem. 2004, 2, 415-420.

15. Plate, R.; Plaum, M. J. M.; Pintar, P.; Jans, C. G.; de Boer, T.; Dijcks, F. A.; Ruigt, G.; Andrews, J. S. Bioorg. Med. Chem. 1998, 6, 1403-1420.

16. Tursun, A.; Aboab, B.; Canet, I.; Sinibaldi, M.-E. Tetrahedron Lett. 2005, 46, 2291-2294.

17. (S)-Solketal was first transformed into its carboxylate potassium salt using $\mathrm{KMnO}_{4}$ in $\mathrm{H}_{2} \mathrm{O} / \mathrm{KOH}$ in $93 \%$ yield according to the procedure described by: Beau, J.-M.; Sinay, P. Tetrahedron Lett. 1985, 26, 6193-6196; Acylation of this salt by $(\mathrm{COCl})_{2}$ in ether with catalytic amounts of pyridine using the method of: Tanaka, A.; Yamashita, K. Agric. Biol. Chem. 1980, 44, 199-202; furnished the attempted $(R)-15$ in $95 \%$ yield.

18. For related processes, see: (a) Ostrowski, J.; Altenbach, H.-J.; Wishnat, R.; Brauer. Eur. J. Org. Chem. 2003, 68, 11041110; (b) Garna, A.; Guidi, A.; Machetti, F.; Menchi, G.; Occhiato, E. G.; Scarpi, D.; Sisi, S.; Trabocchi. J. Org. Chem. 1999, 64, 7347-7364.

19. (a) Sugimoto, T.; Ishihara, J.; Murai, A. Tetrahedron Lett. 1997, 38, 7379-7382; (b) Marco-Contelles, J.; Gallego, P.; 
Rodríguez-Fernández, M.; Khiar, N.; Destabel, C.; Bernabé, M.; Martínez-Grau, A.; Chiara, J. L. J. Org. Chem. 1997, 62, 7397-7412; (c) Wagner, D.; Verheyden, J. P. H.; Moffatt, J. G. J. Org. Chem. 1974, 39, 24-30; (d) David, S.; Hanessian, S. Tetrahedron 1985, 41, 643-663.

20. Azuma, H.; Takao, R.; Niiro, H.; Shikata, K.; Tamagaki, S.; Tachibana, T.; Ogino, K. J. Org. Chem. 2003, 68, 2790-2797.

21. Mukaiyama, T.; Shiina, I.; Iwadare, H.; Saitoh, M.; Nishimura, T.; Ohkawa, N.; Sakoh, H.; Nishimura, K.; Tani, Y.-I.; Hasegawa, M.; Yamada, K.; Saitoh, K. Chem.-Eur. J. 1999, $5,121-161$.
22. Green, M. E.; Rech, J. C.; Floreancig, P. E. Org. Lett. 2005, 7, $4117-4120$.

23. Banwell, M. G.; McRae, K. J. J. Org. Chem. 2001, 66, 67686774.

24. Oikawa, M.; Ueno, T.; Oikawa, H.; Ichihara, A. J. Org. Chem. $1995,60,5048-5068$.

25. (a) Amouroux, A. Heterocycles 1984, 22, 1489-1492; (b) Holzapfel, C. W.; Portwig, M.; Williams, D. B. G. S. Afr. J. Chem. 1999, 52, 165-167; (c) Conway, J. C.; Quayle, P.; Regan, A. C.; Urch, C. J. Tetrahedron 2005, 61, 1191011923. 